\title{
Systematic testing and specificity mapping of alloantigen-specific chimeric antigen receptors in regulatory T cells
}

\author{
Nicholas A.J. Dawson, ${ }^{1,3}$ Caroline Lamarche, ${ }^{2,3}$ Romy E. Hoeppli, ${ }^{2,3}$ Peter Bergqvist, ${ }^{4}$ \\ Vivian C.W. Fung, ${ }^{2,3}$ Emma Mclver, ${ }^{2,3}$ Qing Huang, ${ }^{2,3}$ Jana Gillies, ${ }^{2,3}$ Madeleine Speck, ${ }^{2,3}$ \\ Paul C. Orban,, 2,3 Jonathan W. Bush,, Majid Mojibian, ${ }^{2,3}$ and Megan K. Levings 2,3,6 $^{3,5}$ \\ 'Department of Medicine and 'Department of Surgery, University of British Columbia (UBC), Vancouver, British Columbia, \\ Canada. ${ }^{3} \mathrm{BC}$ Children's Hospital Research Institute (BCCHR), Vancouver, British Columbia, Canada. ${ }^{4}$ Centre for Drug and \\ Research and Development, Vancouver, British Columbia, Canada. ${ }^{5}$ Department of Pathology and Laboratory Medicine and \\ ${ }^{6}$ School of Biomedical Engineering, UBC, Vancouver, British Columbia, Canada.
}

\begin{abstract}
Chimeric antigen receptor (CAR) technology can be used to engineer the antigen specificity of regulatory $T$ cells (Tregs) and improve their potency as an adoptive cell therapy in multiple disease models. As synthetic receptors, CARs carry the risk of immunogenicity, particularly when derived from nonhuman antibodies. Using an HLA-A*02:01-specific CAR (A2-CAR) encoding a single-chain variable fragment $(F v)$ derived from a mouse antibody, we developed a panel of $\mathbf{2 0}$ humanized A2-CARs (hA2-CARs). Systematic testing demonstrated variations in expression, and ability to bind HLA-A*02:01 and stimulate human Treg suppression in vitro. In addition, we developed a new method to comprehensively map the alloantigen specificity of CARs, revealing that humanization reduced HLA-A cross-reactivity. In vivo bioluminescence imaging showed rapid trafficking and persistence of hA2-CAR Tregs in A2-expressing allografts, with eventual migration to draining lymph nodes. Adoptive transfer of hA2-CAR Tregs suppressed HLA-A2+ cell-mediated xenogeneic graft-versus-host disease and diminished rejection of human HLA-A2 ${ }^{+}$skin allografts. These data provide a platform for systematic development and specificity testing of humanized alloantigenspecific CARs that can be used to engineer specificity and homing of therapeutic Tregs.
\end{abstract}

Authorship note: NAJD and CL contributed equally to this work. REH and PB contributed equally to this work.

Conflict of interest: The authors of this manuscript have received research funding from TxCell SA to partially support this work. Patents based on this work have been filed (PCT/CA2018/051167 and PCT/ CA2018/051174)

License: Copyright 2019, American Society for Clinical Investigation.

Submitted: July 18, 2018 Accepted: February 5, 2019 Published: March 21, 2019

\section{Reference information:} JCI Insight. 2019;4(6):e123672. https://doi.org/10.1172/jci. insight.123672.

\section{Introduction}

Tregs hold tremendous promise as an adoptive cell therapy to prevent or treat undesired immune responses in transplantation or autoimmunity (reviewed in ref. 1). Currently, most clinical trials of Treg therapy administer polyclonal Tregs with unknown antigen (Ag) specificity, meaning that large numbers of cells carrying a risk of off-target immunosuppression are required to achieve a therapeutic effect. Consequently, methods to isolate, expand, and/or engineer Ag-specific Tregs is an area of intense interest (2).

An established method of enriching Ag-specific Tregs is through repetitive stimulation in vitro. The feasibility of this approach has been successfully demonstrated in the context of transplantation (3), leading to several ongoing clinical trials to test the safety and efficacy of donor allogeneic Ag-expanded (alloAg-expanded) Tregs in kidney or liver transplantation (1). However, this approach requires complex cell culture and is only feasible because up to $\sim 10 \%$ of Tregs are naturally alloAg-specific (4). We and others have recently shown that an alternate way to generate Ag-specific Tregs is to engineer their specificity using chimeric Ag receptor (CAR) technology. Mouse Tregs engineered to express model-relevant CARs can suppress colitis $(5,6)$ and experimental autoimmune encephalomyelitis (7). Human factor VIII-specific CAR Tregs suppress hemophilia in a humanized mouse model (8), and alloAg-specific human CAR Tregs suppress xenogeneic graft-versus-host disease (GvHD) and skin rejection in xenogeneic mouse models (9-11).

CARs classically contain a single chain of antibody variable domains ( $\mathrm{scFv}$ ) derived from heavy and light chains of well-characterized, high-affinity $\mathrm{mAbs}$, which face the extracellular space (12). CARs derived from mouse scFvs can be highly effective (13-16) but carry the risk of immunogenicity, with sensitization possibly limiting therapeutic efficacy and repeat dosing (17-23). Therapeutic mAb studies have 
shown that the structural residues in mouse variable regions are sufficient to elicit immune responses ( 24 , 25 ), leading to bioinformatic strategies to "humanize" mAbs (26-29) so that $>90 \%$ of the structure originates from human, with only the Ag-binding complementarity-determining region (CDR) remaining from mouse. This approach can be highly successful, as evidenced by the numerous humanized mAbs in routine clinical use (30), but is empirical and can result in decreased affinity and loss or change of specificity $(26,31)$. The applicability of bioinformatic strategies developed to humanize mAbs to create humanized CARs is largely unknown.

In addition to immunogenicity, a specific consideration for alloAg-specific mAbs is that because many HLA alleles differ by only a few amino acids (32), there is often cross-reactivity with closely related HLA proteins, with anti-HLA mAbs recognizing multiple alleles within an evolutionarily related family. Cross-reactivity of anti-HLA mAbs/CARs could be problematic for a CAR Treg approach, as exquisite specificity for the donor organ is needed to prevent the risk of systemic CAR Treg activation by cross-reactive HLA alleles expressed by the recipient.

Using our previously described HLA-A*02:01-specific CAR, which was derived from the scFv of the mouse BB7.2 mAb (9), we report an effective way to generate multiple humanized CARs. In addition to systematic testing for in vitro and in vivo function in Tregs, we developed a methodology to comprehensively determine HLA specificity. This approach can be used as a platform to generate a series of HLA-specific CARs to advance the use of CAR Tregs as a widely applicable therapy to prevent allograft rejection.

\section{Results}

Design and expression of a panel of humanized A2-CARs. The amino acid sequences of the variable regions of the heavy and light chains from the mouse BB7.2 mAb were aligned to the human immunoglobulin sequences obtained from the international ImMunoGeneTics (IMGT) information system database using IgBLAST (National Center for Biotechnology Information). The V-gene delimitation system was set to the Kabat sequences to obtain the Kabat-defined CDRs (33). In addition, the Chothia definition (34) was determined.

A number of different human germline genes were identified as possible framework sequences, and we selected those that were most homologous to the mouse sequence and encoded CDRs with lengths similar to those in BB7.2 for CDR grafting. The human CDRs from these candidate human germline genes were replaced with the mouse counterpart CDRs from BB7.2 using the Chothia and Kabat numbering systems $(33,34)$. Ultimately, 6 humanized heavy chains based on 4 human germline V-genes, and 5 humanized light chains based on 5 human germline V-genes were generated, resulting in 18 humanized CARs generated by combining different humanized heavy and light chains (Figure 1A).

Humanization can affect Ag specificity $(26,31)$, so we first transiently transfected humanized A2-CARs (hA2-CARs) into 293T cells and used HLA-A*02:01 tetramers to quantify Ag binding by flow cytometry. Of the $18 \mathrm{hA}$-CAR constructs tested, only 10 were expressed and bound to the $\mathrm{A}^{*} 02: 01$ tetramer (Supplemental Figure 1; supplemental material available online with this article; https://doi.org/10.1172/jci. insight.123672DS1; and Table 1). To test expression and function of these $10 \mathrm{hA2}$-CARs in Tregs, CD4 ${ }^{+} \mathrm{C}-$ $\mathrm{D} 25^{\mathrm{hi}} \mathrm{CD} 127^{\mathrm{lo}}$ cells were transduced with the hA2-CARs, the original mouse A2-CAR (mA2-CAR) (9), or a vector-only control encoding the truncated nerve growth factor receptor ( $\triangle \mathrm{NGFR}$ ) transduction marker but no CAR. Following transduction and expansion, CAR Tregs retained high expression of FOXP3 (Supplemental Figure 2). CAR cell-surface expression and specificity were tested by staining with an $A^{*} 02: 01$ tetramer, revealing strong, uniform binding to $7 \mathrm{hA} 2$-CARs and low, bimodal binding to 3 (H2k2, H4k4, and H5k4) hA2-CARs (Figure 1B and Table 1). In addition, although the H4k3 hA2-CAR was expressed on a similar proportion of Tregs, it was expressed at a lower MFI than the mA2-CAR.

Treg activation potential of a panel of hA2-CARs. We next tested the ability of the $10 \mathrm{hA2}$-CAR variants to activate Tregs. Tregs expressing one of the $10 \mathrm{hA2}-\mathrm{CAR}$ variants, $\mathrm{mA2}-\mathrm{CAR}$, or $\triangle \mathrm{NGFR}$ alone were stimulated via the CAR (Figure 2), left unstimulated, or stimulated via T cell receptor (TCR) (Supplemental Figure 3). Stimulation of $\mathrm{m} / \mathrm{hA} 2-\mathrm{CAR}$ Tregs with A2-expressing artificial Ag-presenting cells (APCs) resulted in high expression of both CD69 and CD71 for most constructs, with the exception of the 3 poorly expressed CARs. Specifically, H2k2 showed low basal activation and a moderate increase in CD69 and CD71 expression after CAR stimulation, whereas $\mathrm{H} 4 \mathrm{k} 4$ and $\mathrm{H} 5 \mathrm{k} 4$ showed high basal activation and no increase in CD69 and CD71 expression when stimulated through the CAR (Figure 2A and Supplemental Figure 3).

Upregulation of proteins associated with Treg function (cytotoxic $\mathrm{T}$ lymphocyte-associated protein 4 [CTLA-4] and latency-associated peptide [LAP]) displayed a similar pattern, with low CAR-stimulated 
A

Murine-dervied CAR construct

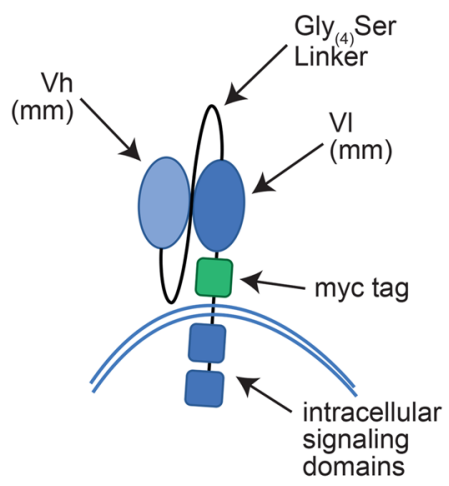

B
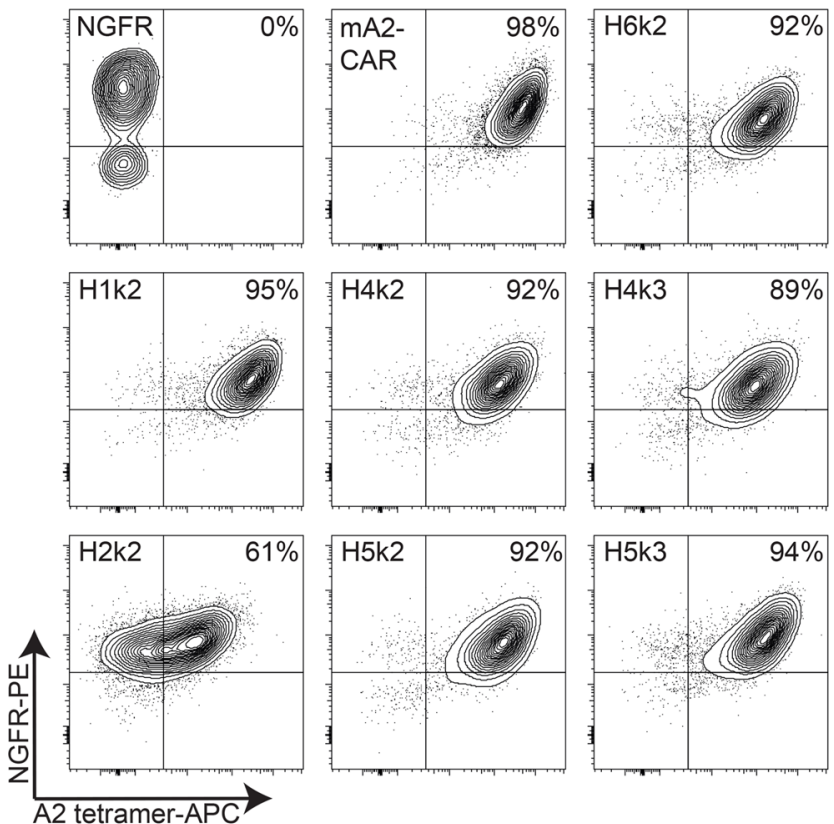

"Humanized" CAR constructs

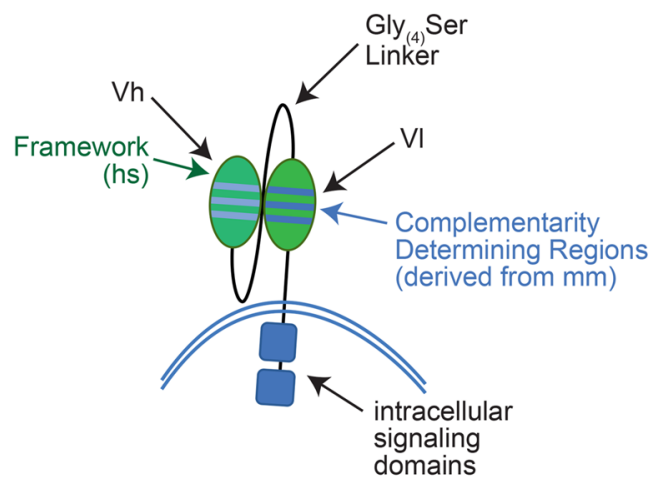

$\begin{array}{ccc}\text { k1 variants } & \text { k3 variants } & \text { k5 variants } \\ \text { H1k1 } & H 3 k 3 & H 1 \text { k5 } \\ \text { k2 variants } & H 4 k 3 & H 3 k 5 \\ \text { H1k2 } & \text { H5k3 } & \text { H4k5 } \\ \text { H2k2 } & \text { k4 variants } & H 5 k 5 \\ \text { H3k2 } & \text { H1k4 } & \\ \text { H4k3 } & \text { H3k4 } & \\ \text { H5k2 } & \text { H4k4 } & \\ \text { H6k2 } & \text { H5k4 } & \end{array}$
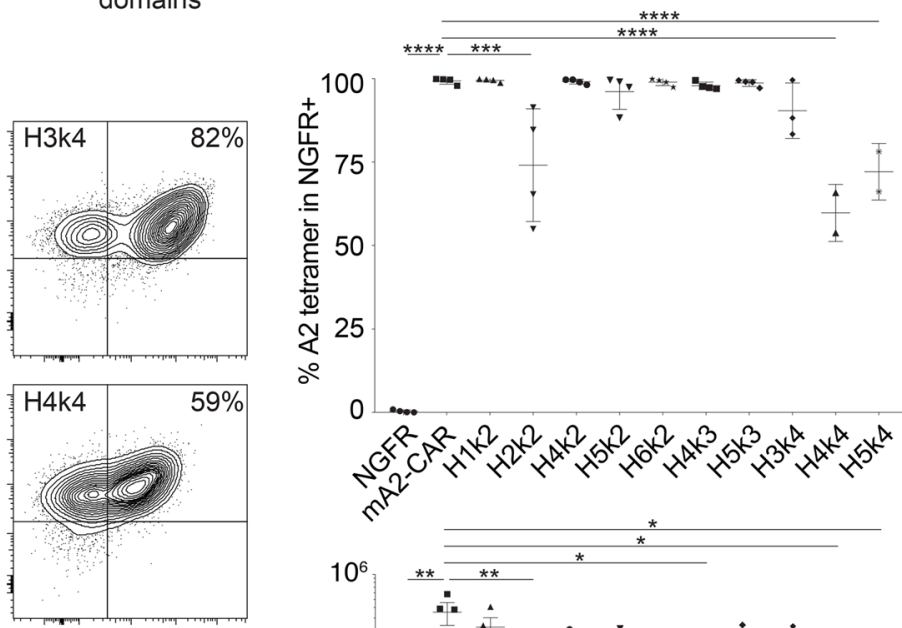

0 -
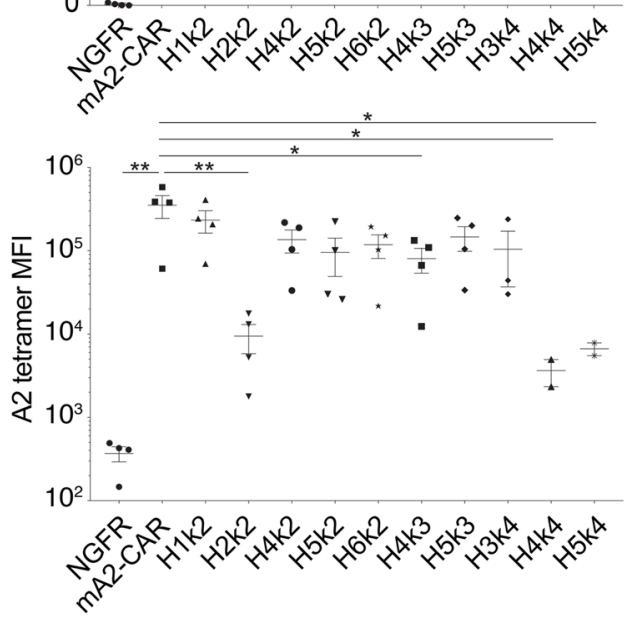

Figure 1. Expression of a panel of hA2-CARs on human Tregs. (A) Schematic representation of CAR humanization. CDRs from the BB7.2-derived scFv were determined using Kabat or Chothia definitions for each heavy and light chain and grafted onto suitable human framework sequences. mm, Mus musculus; hs, Homo sapiens. (B) Human Tregs were transduced with lentivirus encoding the indicated constructs. After 7 days of expansion, the ability of $\triangle \mathrm{NGFR}^{+}$cells to bind to HLA-A*02:01 tetramers was measured by flow cytometry. Left: Representative flow cytometry plots. Right: Summarized data of percent or MFI of $A^{*} 02: 01$-tetramer binding. Data represent $n=2-4$ for each construct pooled from at least 2 independent experiments. Mean \pm SEM. ${ }^{*} P<0.05,{ }^{* *} P<0.01,{ }^{* *} P<0.001,{ }^{* * *} P<0.0001$.

expression of CTLA-4 and LAP for H2k2, H4k4, and H5k4 (Figure 2B). Similar to CD69 and CD71, H4k 4 and H5k4 had low CAR-stimulated upregulation of CTLA-4 and LAP compared with unstimulated controls. These data suggest that the impaired activation capacity of H4k4 and H5k4, but not H2k2, may be driven by tonic CAR signaling, an interpretation supported by evidence that TCR-mediated activation is also hampered in cells expressing these constructs (Supplemental Figure 3B).

Alloantigen specificity mapping of hA2-CAR constructs. T cell alloAg specificity is traditionally tested by in vitro stimulation with PBMCs of known haplotypes. This approach, however, is cumbersome and imprecise, as it requires an extensive bank of viable, haplotyped PBMCs, and specificity toward individual HLA alleles cannot be determined in isolation. We sought to develop a more feasible, comprehensive, and accurate way to assess the cross-reactivity of alloAg-specific CARs. Specifically, we hypothesized that the One Lambda Flow Panel Reactive Antibody (FlowPRA) single Ag beads previously developed to measure 
serum alloantibody titers (35), which consist of fluorescently labeled beads coupled to single HLA Ags, could be adapted to measure alloAg-directed CAR Treg specificity. Tregs expressing the $\mathrm{m} / \mathrm{hA} 2$-CARs or $\triangle$ NGFR were incubated with a mixture of FlowPRA Single Antigen beads, then analyzed by flow cytometry using forward scatter/side scatter (FSC/SSC) to identify beads, cells, and bead/cell conjugates (hereafter called the FlowPRT cell assay). Since the number of beads bound per cell and cells bound per bead could not be controlled, the amount of CAR Treg binding to beads was assessed by comparing the number of unbound beads in samples incubated with CAR Tregs versus the $\triangle$ NGFR Treg control (see Methods for formula calculation; Figure 3A).

To validate the methodology, the relative binding of each $\mathrm{m} / \mathrm{hA2}-\mathrm{CAR}$ construct to HLA-A*02:01 as determined by the FlowPRT cell assay (Figure 3B) was compared with the MFI of tetramer binding (Figure $3 C)$. This analysis revealed a strong, direct correlation between the two methods of detecting $A^{*} 02: 01$ binding. The data also revealed that $\mathrm{H} 2 \mathrm{k} 2$ and $\mathrm{H} 4 \mathrm{k} 4$ were less able to bind to $\mathrm{A}^{*} 02: 01$ and a trend toward low binding with $\mathrm{H} 5 \mathrm{k} 4$, consistent with their low expression and activation capacity. We further asked whether the amount of $\mathrm{A}^{*} 02: 01$ binding, as determined by the FlowPRT cell assay, correlated with the biological effect of exposure to $A^{*} 02: 01$. Indeed, we found there was a direct correlation between the amount of $A^{*} 02: 01$ binding quantified by the FlowPRT cell assay and stimulation of Treg activation, as judged by CD69 upregulation following exposure to $A^{*} 02: 01$-expressing APCs (Figure 3D). These data demonstrate the utility of the FlowPRT cell method for measuring the ability of alloAg-specific CARs to bind to different HLA alleles.

We next examined the degree of $\mathrm{m} / \mathrm{hA} 2-\mathrm{CAR}$ Treg cross-reactivity to alleles of HLA-A and HLA-B. BB7.2 has been reported to bind to HLA-A*23:01, $A * 24: 02$, and $A * 69: 01$ (36). When we tested BB7.2 binding in the single Ag FlowPRA assay, we confirmed high binding to $A * 69: 01$ but could not confirm cross-reactivity to $A^{* 23: 01}$ or $A * 24: 02$ (Supplemental Figure 4 ). We next tested the relative ability of the $\mathrm{m} / \mathrm{hA2}-\mathrm{CAR}$ Tregs to bind to various HLA-A alleles. We found the mA2-CAR Tregs bound significantly

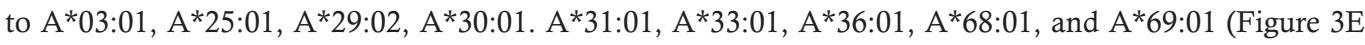
and Supplemental Table 1). In contrast, all variants of hA2-CAR Tregs displayed reduced cross-reactivity compared with mA2-CAR Tregs. As expected, all CAR constructs bound to A*69:01, a variant of $A^{*} 02: 01$ differing by only 6 amino acids, which is also bound by BB7.2. None of the CAR constructs displayed any significant binding to HLA-B (Supplemental Figure 5).

Biological effects of cross-reactive HLA-A alleles. The relationship between the degree of CAR Treg Ag binding and biological activity is unknown. To define the biological significance of HLA cross-reactivity, we generated APCs expressing HLA-A*24:02, $-A^{*} 25: 01,-A * 68: 01$, or $-A * 69: 01$. We found that only coculture with HLA-A*02:01- or HLA-A*69:01-expressing cells resulted in activation of $\mathrm{m} / \mathrm{hCAR}$ Tregs, as judged by upregulated expression of CD69, CD71, LAP, CTLA-4 (Figure 4), or CD40L (data not shown). The lack of stimulation by HLA-A*24:02, $-A^{*} 25: 01$, or $-A^{*} 68: 01$-expressing K562 cells was not due to poor HLA expression (Supplemental Figure 6). These data suggested that effective CAR-mediated activation of Tregs requires high-affinity and/or -avidity interactions. Accordingly, while some hA2-CARs showed binding to $A *$ 25:01 and $A * 68: 01$ in the FlowPRT assay, the strength of binding was insufficient for cellular activation.

hA2-CAR Tregs are suppressive in vitro and in vivo. We used mixed lymphocyte reactions (MLRs) with $\mathrm{A}^{*}$ 02:01-expressing dendritic cells to test the ability of the hA2-CAR constructs to stimulate Treg suppressive function. The 3 poorly functional constructs $(\mathrm{H} 2 \mathrm{k} 2, \mathrm{H} 4 \mathrm{k} 4$, and $\mathrm{H} 5 \mathrm{k} 4)$ were excluded. Proliferation of third-party HLA-A*02:01 ${ }^{\text {neg }}$ PBMCs was stimulated by coculture with mature HLA-A*02:01 ${ }^{\text {pos }}$ monocyte-derived dendritic cells in the absence or presence of increasing ratios of $\mathrm{m} / \mathrm{hA2}$-CAR Tregs or control Tregs. Both mA2- and hA2-CAR-expressing Tregs were significantly better at suppressing alloAg-stimulated proliferation of $\mathrm{CD}^{+} \mathrm{T}$ cells in comparison to control Tregs transduced with the $\triangle \mathrm{NGFR}$ control up to a ratio of 1:16 Tregs/PBMCs (Figure 5A).

To confirm the functional capacity of hA2-CAR Tregs in vivo, we used a model of xenogeneic GvHD in which we previously showed that HLA-A2-specific CAR Tregs were more potent than nonspecific (polyclonal) CAR Treg controls (9). Using this model, we compared one of the 6 hA2-CARs that displayed optimal characteristics (Table 1), H1k2, with the original mA2-CAR construct. HLA-A*02:01 ${ }^{\text {pos }}$ PBMCs were injected into NSG mice with or without H1k2-CAR Tregs, or mA2-CAR Tregs as a positive control. Mice that received $\mathrm{mA2}$ - or hA2-CAR-expressing Tregs had significantly improved survival and reduced human $\mathrm{CD}^{+} 5^{+}$ cell engraftment compared with those that did not receive CAR Tregs (Figure 5, B-D, and Supplemental Figure 7A). While the biological effect of the Tregs was observed, as in our previous study (9), we did not detect circulating m/hA2-CAR Tregs, measured from 14 days after injection (Supplemental Figure 7B). 
Table 1. Summary of hA2-CAR Treg construct performance in various in vitro assays

\begin{tabular}{|c|c|c|c|c|c|}
\hline hA2-CAR variant & $\begin{array}{l}\text { HLA-A*02:01 binding } \\
\text { (293T cells) }\end{array}$ & $\begin{array}{c}\text { HLA-A*02:01 binding } \\
\text { (Tregs) }\end{array}$ & $\begin{array}{c}\text { HLA-A }{ }^{*} \text { 02:01-mediated } \\
\text { activation }\end{array}$ & $\begin{array}{l}\text { Low cross-reactivity to } \\
\text { other HLA-A alleles }\end{array}$ & $\begin{array}{c}\text { Antigen-specific } \\
\text { suppression (MLR) }\end{array}$ \\
\hline H1k1 & X & - & - & - & - \\
\hline $\mathrm{H} 1 \mathrm{k} 2$ & $\sqrt{ }$ & $\sqrt{ }$ & $\sqrt{ }$ & $\sqrt{ }$ & $\sqrt{ }$ \\
\hline $\mathrm{H} 2 \mathrm{k} 2$ & $\sqrt{ }$ & $x$ & $x$ & $\sqrt{ }$ & - \\
\hline $\mathrm{H} 3 \mathrm{k} 2$ & $x$ & - & - & - & - \\
\hline $\mathrm{H} 4 \mathrm{k} 2$ & $\sqrt{ }$ & $\sqrt{ }$ & $\sqrt{ }$ & $\sqrt{ }$ & $\sqrt{ }$ \\
\hline $\mathrm{H} 5 \mathrm{k} 2$ & $\sqrt{ }$ & $\sqrt{ }$ & $\sqrt{ }$ & $\sqrt{ }$ & $\sqrt{ }$ \\
\hline $\mathrm{H} 6 \mathrm{k} 2$ & $\sqrt{ }$ & $\sqrt{ }$ & $\sqrt{ }$ & $\sqrt{ }$ & $\sqrt{ }$ \\
\hline H3k3 & $x$ & - & - & - & - \\
\hline $\mathrm{H} 4 \mathrm{k} 3$ & $\sqrt{ }$ & $\sqrt{ } / X$ & $\sqrt{ }$ & $\sqrt{ }$ & $\sqrt{ }$ \\
\hline H5k3 & $\sqrt{ }$ & $\sqrt{ }$ & $\sqrt{ }$ & $\sqrt{ }$ & $\sqrt{ }$ \\
\hline $\mathrm{H} 1 \mathrm{k} 4$ & $x$ & - & - & - & - \\
\hline $\mathrm{H} 3 \mathrm{k} 4$ & $\sqrt{ }$ & $\sqrt{ }$ & $\sqrt{ }$ & $\sqrt{ }$ & $\sqrt{ }$ \\
\hline $\mathrm{H} 4 \mathrm{k} 4$ & $\sqrt{ }$ & $x$ & $x$ & $\sqrt{ }$ & - \\
\hline $\mathrm{H} 5 \mathrm{k} 4$ & $\sqrt{ }$ & $x$ & $x$ & $\sqrt{ }$ & - \\
\hline $\mathrm{H} 1 \mathrm{k} 5$ & $x$ & - & - & - & - \\
\hline H3k5 & $x$ & - & - & - & - \\
\hline $\mathrm{H} 4 \mathrm{k} 5$ & $x$ & - & - & - & - \\
\hline H5k5 & $X$ & - & - & - & - \\
\hline
\end{tabular}

hA2-CAR Tregs traffic to HLA-A2 ${ }^{+}$skin grafts in vivo. For effective suppression of allograft rejection, Tregs need to migrate to the allograft and control local immunity $(37,38)$. To test how CAR-directed specificity affected Treg trafficking, we performed side-by-side skin transplants from NSG or NSG-A*02:01-transgenic mice onto NSG mice (Figure 6A). After graft recovery, PBMCs were injected in the absence or presence of $\mathrm{m} / \mathrm{hA2}$-CAR Tregs or with HER2-CAR Tregs as a nonspecific polyclonal Treg control (9). In addition to the CAR, Tregs were cotransduced with a lentivirus encoding a luciferase-GFP fusion protein. Bioluminescence imaging was performed after D-luciferin injection up to 21 days after Treg injection (39). In contrast to polyclonal HER2-CAR Tregs, which trafficked equally to A2-negative and -positive skin, m/hA2CAR Tregs rapidly trafficked to the A2-expressing skin. In addition, $\mathrm{m} / \mathrm{hA2}$-CAR Tregs persisted longer than nonspecific HER2-CAR Tregs. Whereas HER2-CAR Tregs were undetectable by day 7-14, a strong $\mathrm{m} / \mathrm{hA2}$-CAR Treg signal remained within the A2-positive skin throughout the experiment (Figure 6B). Quantification of the ratio of luminescence in the A2-positive versus A2-negative graft revealed significant Ag-driven trafficking of both H1k2- and mA2-CAR Tregs to the A2-expressing graft (Figure 6, C and D).

In addition to graft-localized $\mathrm{m} / \mathrm{hA} 2-\mathrm{CAR}$ Tregs, we noted an adjacent signal consistent with the location of a local draining lymph node. The timing of when this signal was first detected was variable, ranging from 7 to 14 days (Figure 6B), and in some mice it waned over time (Supplemental Figure 8A). In a subset of animals, flow cytometric analysis of skin graft draining lymph nodes revealed a substantial proportion of $\mathrm{hCD}^{+} \mathrm{FOXP}^{+} \Delta \mathrm{NGFR}^{+} \mathrm{A} 2$-tetramer ${ }^{+} \mathrm{CAR}$ Tregs. In contrast, very few of these cells were detected in the spleen (Supplemental Figure 8, B-D).

hA2-CAR Tregs prevent human skin allograft rejection. To evaluate the immunoregulatory potential of hA2-CAR Tregs in a solid organ transplant model, we used a humanized model of skin transplantation in which NSG mice were transplanted with human HLA-A2 ${ }^{\text {pos }}$ skin grafts. After 6 weeks, mice were injected with HLA-A2 ${ }^{\text {neg }}$ PBMCs with or without autologous $\triangle$ NGFR Tregs or H1k2 hA2-CAR Tregs. Four weeks after cell injection, mice were sacrificed, and the skin graft was collected for evaluation of pathology and inflammatory cytokine expression. All mice maintained stable body weight, indicating a lack of xenogeneic GvHD (Figure 7A), with evidence of human leukocyte engraftment in blood and spleen (Figure 7B). H\&E sections were evaluated for rejection in a blinded manner by a pathologist using a 25-point scale, revealing a significant decrease in the cumulative pathological rejection score in mice that received $\mathrm{H} 1 \mathrm{k} 2 \mathrm{hA} 2-\mathrm{CAR}$ versus PBMCs alone (Figure 7C). Immunostaining revealed that, in comparison to mice receiving PBMCs alone, mice receiving PBMCs and H1k2 hA2-CAR Tregs had a 
A
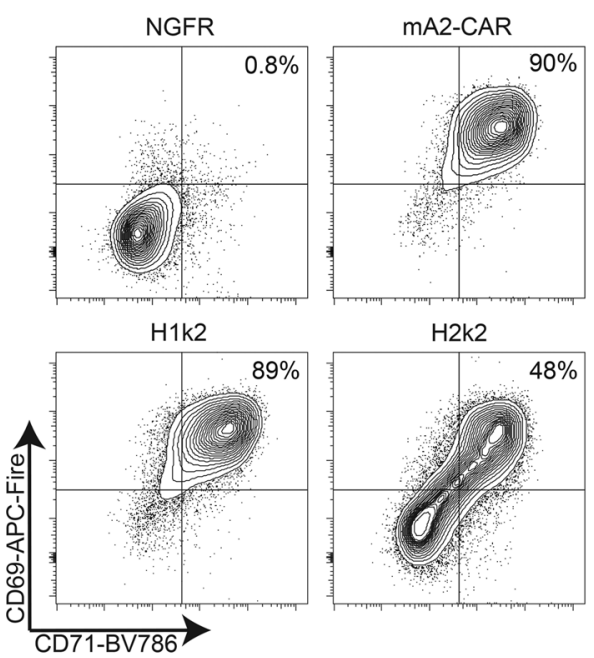

$\mathrm{H} 2 \mathrm{k} 2$

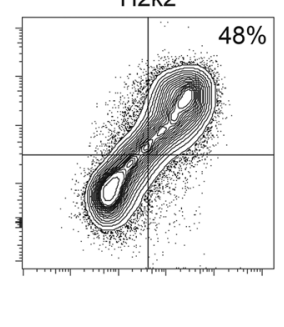

B

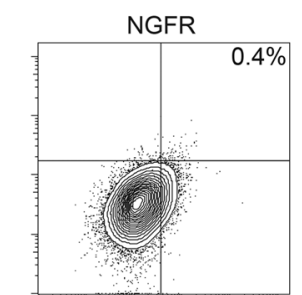

$\mathrm{H} 1 \mathrm{k} 2$

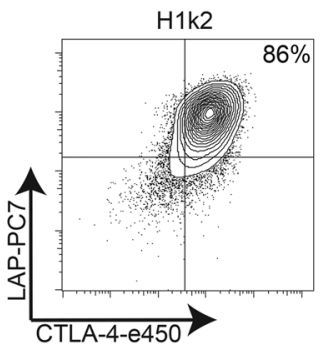

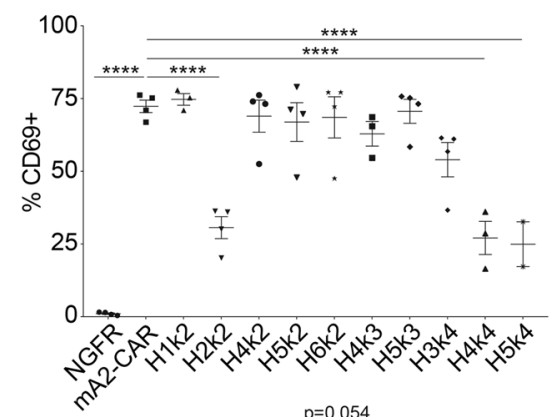
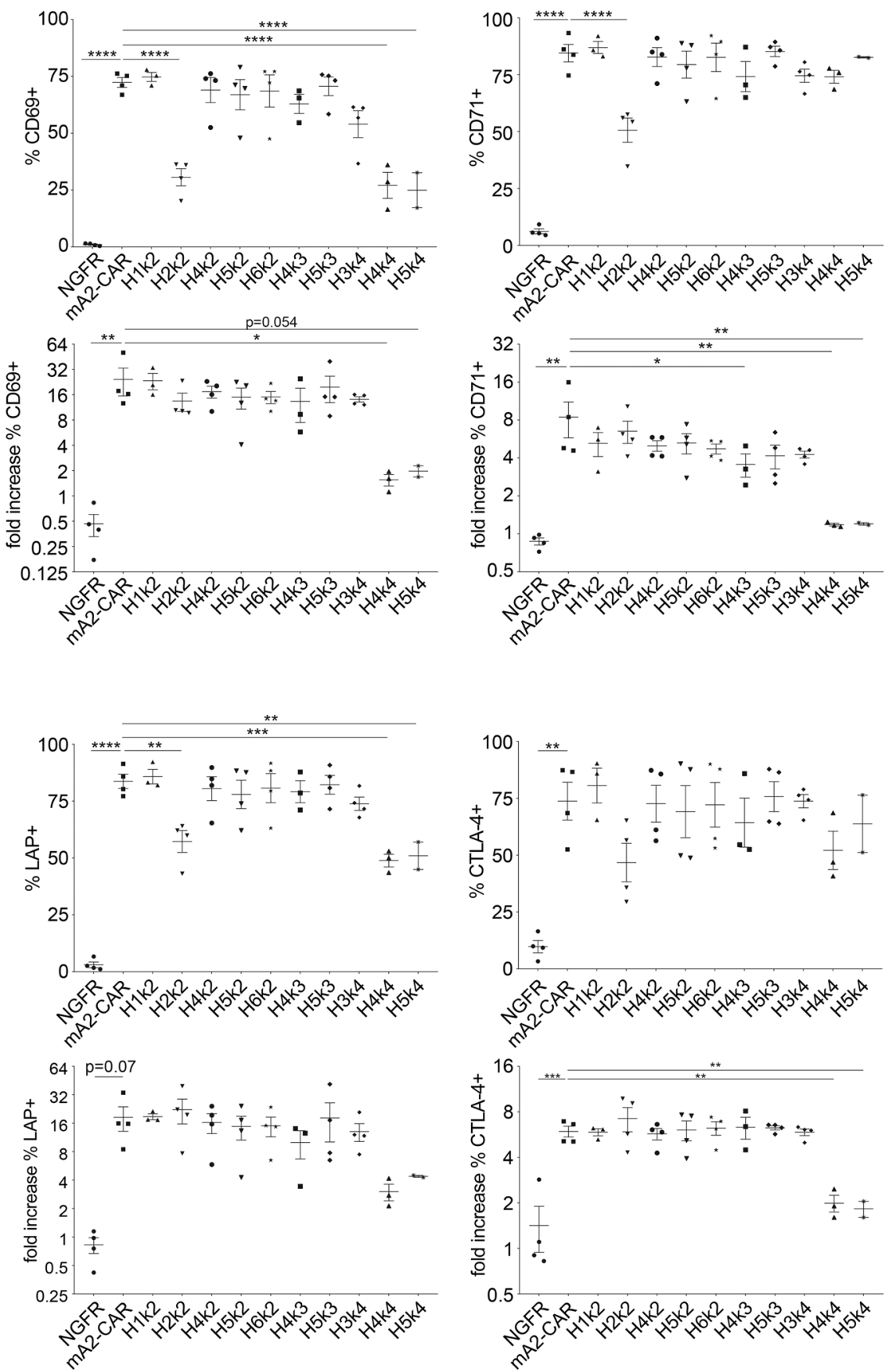

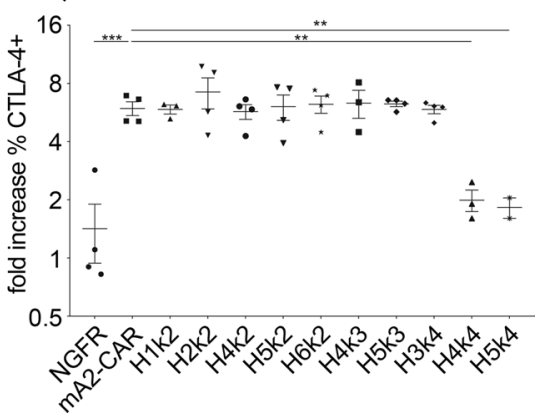

Figure 2. In vitro function of a panel of hA2-CARs on human Tregs. (A and B) $\triangle N G F R$ control/CAR Tregs were cocultured with a 2:1 (Tregs: K562) ratio of HLA-A2-expressing K562 cells. After 16 hours, expression of CD69, CD71, CTLA-4, and LAP was measured by flow cytometry. (A) Percent positive and fold increase over baseline (no K562; Supplemental Figure 3B) expression of CD69 and CD71. (B) Percent positive and fold increase over baseline (no K562; Supplemental Figure 3B) expression of CTLA-4 and LAP. Data represents $n=2-4$ for each construct pooled from at least 2 independent experiments. One-way ANOVA and Holm-Šídák post hoc test comparing all constructs with mA2-CAR Tregs. Mean \pm SEM. ${ }^{*} P<0.05,{ }^{* *} P<0.01,{ }^{* * *} P<0.001,{ }^{* * * *} P<0.0001$.

significant reduction in $\mathrm{Ki}-67^{+}$keratinocytes, and a trend toward reduced involucrin destruction (Figure 7D). Quantitative PCR (qPCR) quantification also showed a general reduction in several inflammatory cytokines within the grafts of $\triangle$ NGFR Treg- or H1k2 hA2-CAR Treg-treated mice (Figure 7E).

Consistent with data from the xenogeneic GvHD model, while PBMCs were detectable in blood from day 14 to 28, CAR Tregs were not (Supplemental Figure 9, A and B). However, immunostaining revealed that in comparison to mice receiving PBMCs alone, mice receiving $\triangle$ NGFR Tregs or H1k2 hA2-CAR Tregs had a trend toward higher proportions of $\mathrm{FOXP}^{+}$cells in the graft (Figure $7 \mathrm{~F}$ ). 
A

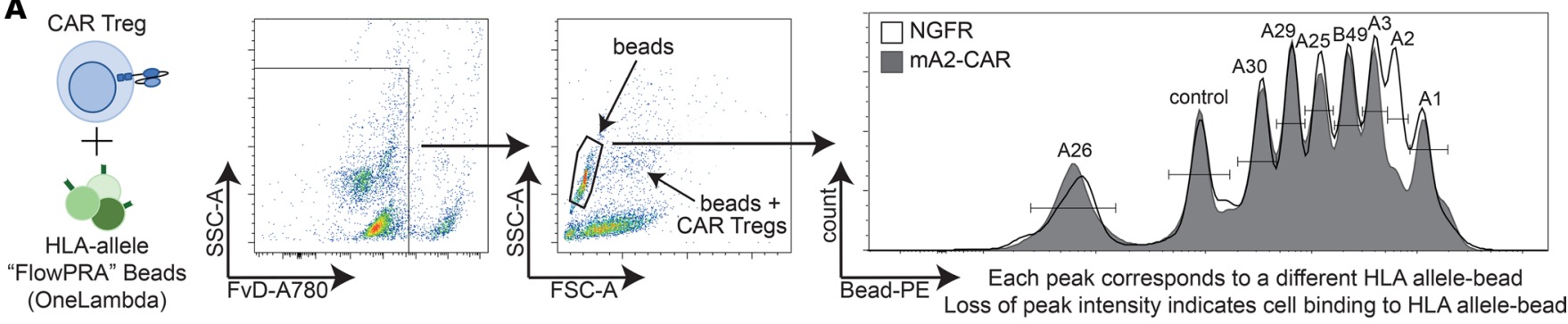

B
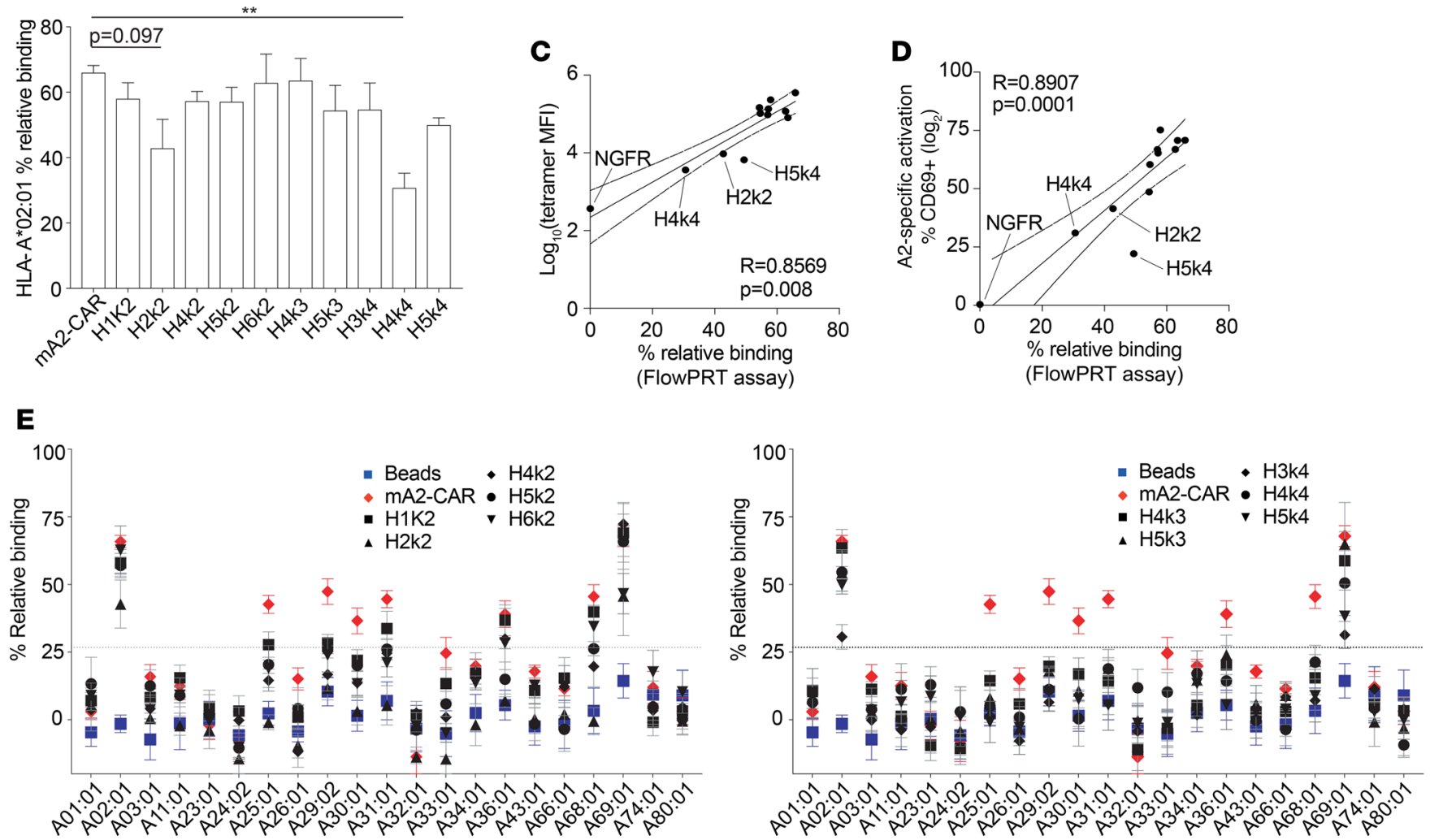

Figure 3. Cross-reactivity of humanized anti-HLA-A2 CARs with common HLA-A allelic variants. (A) Schematic diagram of experimental setup and gating strategy for the FlowPRT cell assay. $\triangle$ NGFR or CAR Tregs were incubated with a cocktail of single HLA FlowPRA beads for 30 minutes, and bead-CAR Treg interactions were quantified as the loss of beads in a bead singlet gate based on FSC/SSC profile. (B) Binding to HLA-A*02:01-coated beads for each $\mathrm{m} / \mathrm{hA2}$-CAR Treg relative to binding of a $\triangle$ NGFR Treg control. Statistical significance determined by 1-way ANOVA and Holm-Šídák post hoc test comparing with $\mathrm{mA2}$-CAR; mean $\pm \mathrm{SEM}$; ${ }^{*} P<0.01$. (C and $\mathbf{D}$ ) Correlation between the mean of HLA-A*02:01 binding measured by the FlowPRT cell assay and either (C) HLA-A*02:01 tetramer MFI evaluated by flow cytometry or (D) increase in the proportion of CD69+ cells 16 hours after coculture with HLA-A*02:01 versus negative control HLA-A*24:01 K562 cells. (E) Percent binding of each m/hA2-CAR Treg to the indicated HLA-A alleles after normalization to an $\triangle$ NGFR Treg control from the same donor. Dotted line represents 2 SDs from the mean of the bead-only control. For a summary of statistical results in $\mathbf{E}$, see Supplemental Table 1. $n=3-6$ from at least 3 independent experiments.

The origin of these skin-localized FOXP3 ${ }^{+}$cells was likely a combination of skin-resident cells (Supplemental Figure 9C), injected PBMCs, and/or the transferred Tregs. The presence of FOXP3 ${ }^{+}$cells was unique to the transplanted skin graft, as they were undetectable in the intestine, liver, or lung (Figure 7G). These data suggest that, as for the model with A2-transgenic NSG skin, H1k2 hA2-CAR Tregs specifically trafficked to human $\mathrm{A} 2^{+}$skin allograft, where they persisted.

\section{Discussion}

Mouse-derived CARs can be highly effective, but $\mathrm{T}$ cells expressing humanized or fully human CARs have increased cell persistence and efficacy (40), and decreased side effects $(17,19-22,41)$. To date, there has been no comprehensive investigation of the applicability of bioinformatic methods for humanizing mAbs for use in CAR constructs. Herein, we describe a method to generate a panel of humanized CARs and 

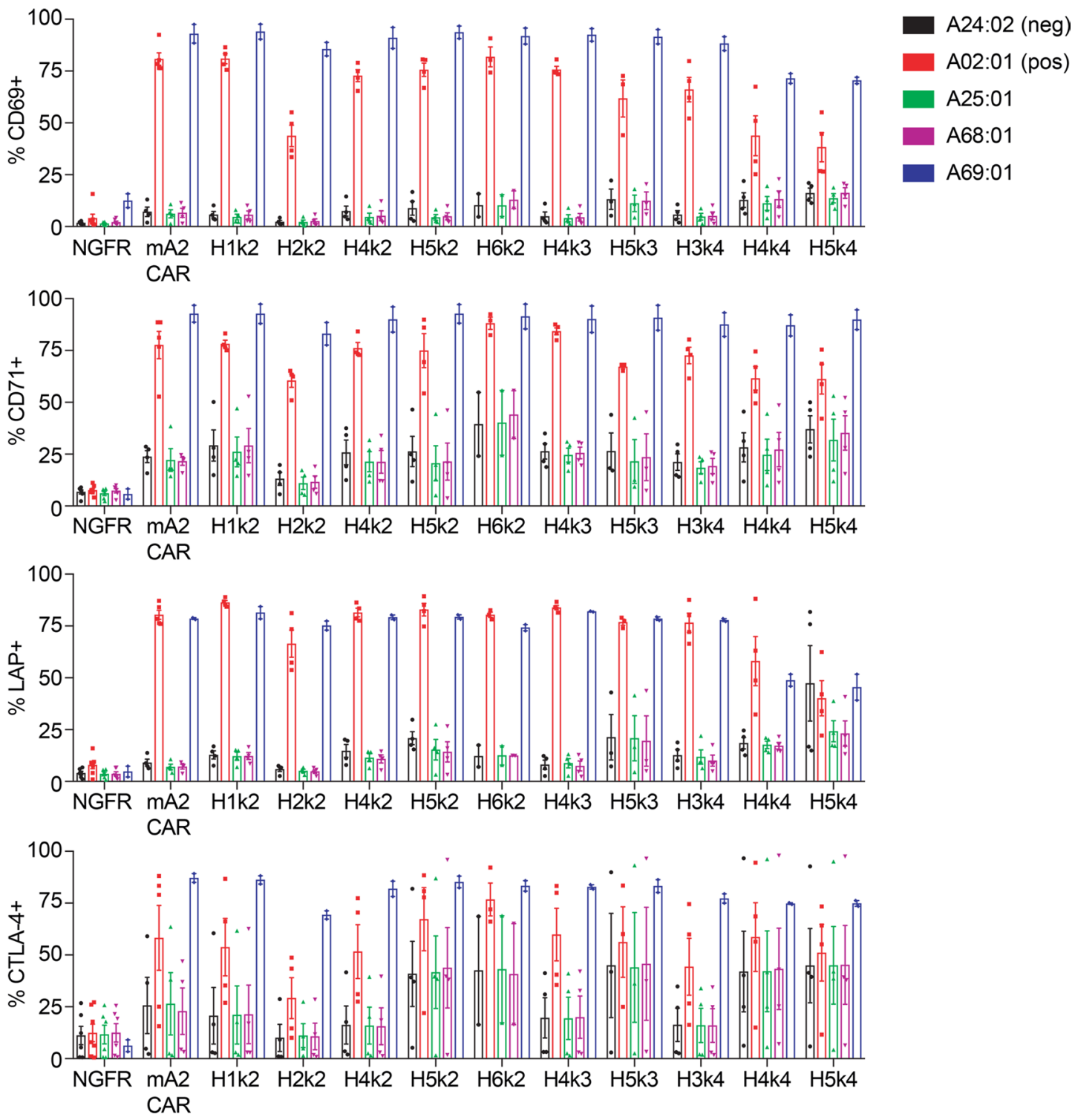

Figure 4. Functional cross-reactivity of a panel of hA2-CAR constructs in human Tregs. $\triangle$ NGFR or m/hA2-CAR Tregs were cocultured with K562 cells transduced to express the indicated HLA-A alleles. After 16 hours, expression of CD69, CD71, LAP, and CTLA-4 was measured on live CD4 ${ }^{+} T$ cells. $n=2-6$ pooled from at least 2 independent experiments, mean \pm SEM.

illustrate the importance of testing multiple versions with a series of assays to identify constructs with optimal specificity and activation capacity, and in vivo function. We also developed a new way to systematically test the specificity of CARs for alloAgs, creating a new platform to comprehensively identify constructs with defined allele specificity.

Of the 18 in silico generated hA2-CARs, 10 constructs maintained specificity for HLA-A*02:01, as judged by tetramer staining in 293T cells. These data are consistent with reports that humanization of scFvs can alter affinity and/or Ag specificity $(42,43)$. Notably, 3 constructs (H2k2, H4K4, and H5k4) bound to tetramer in $293 \mathrm{~T}$ cells but, when expressed in human Tregs, displayed a significantly reduced ability to bind to $\mathrm{A}^{*} 02: 01$, both in terms of percent tetramer ${ }^{+}$cells and MFI. Their bimodal expression pattern suggestive of receptor internalization, high basal activation levels, and failure to become further activated in the presence of CAR or TCR stimulation, suggests that these CARs may induce "tonic" activation in Tregs. Interestingly, evidence of functional tonic activation was not uniformly revealed with all activation markers tested. Specifically, for all 3 constructs, functional tonic activation was clearly evident with CD69 and LAP but not with CTLA-4; for CD71, it was only evident for H2k2. Thus, when screening a panel 
A
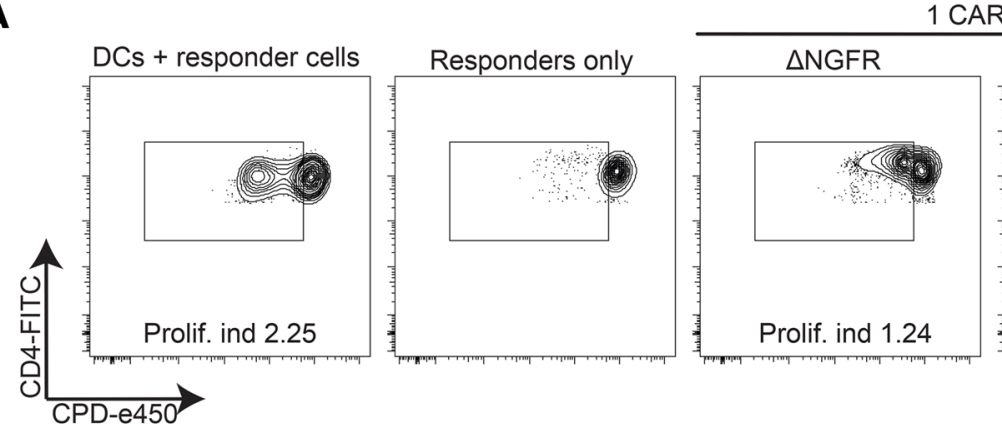

1 CAR Treg : 1 PBMC responder cell
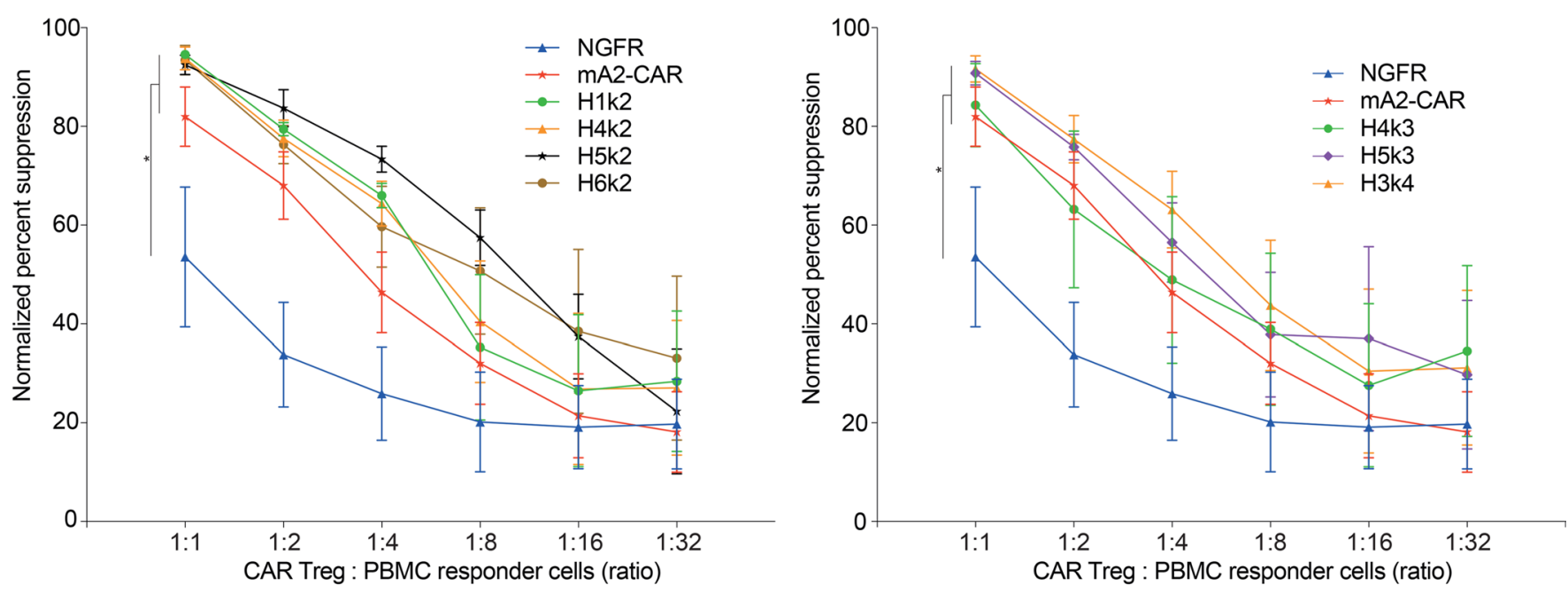

B

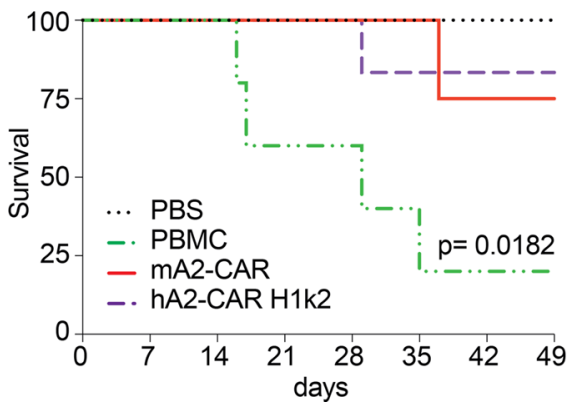

C

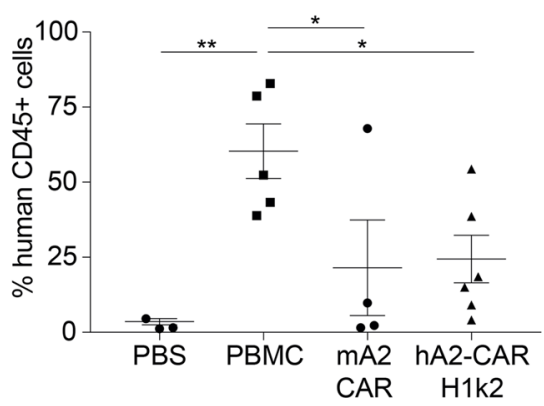

D

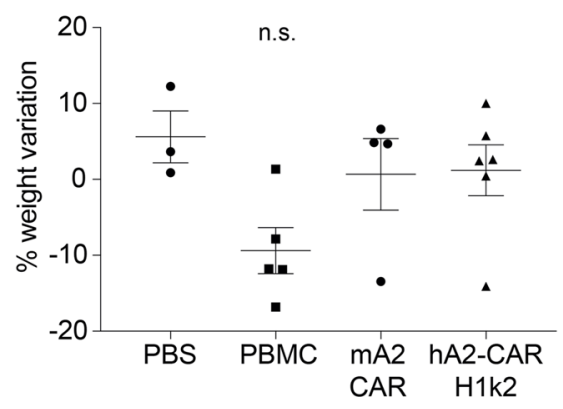

Figure 5. hA2-CAR Tregs are suppressive in vitro and in a model of xenogeneic GvHD in vivo. (A) Cell proliferation dye-e450-labeled (CPD-e450labeled) HLA-A2 ${ }^{\text {neg }} C D 4^{+}$"responder" $T$ cells were stimulated with a 1:1 ratio of mature HLA-A2 ${ }^{+}$dendritic cells in the absence/presence of varying ratios of the indicated CPD-e660-labeled control or m/hA2-CAR Tregs. After 6 days, the amount of proliferation of the CPD-e450-labeled CD4+ responder T cells was measured by flow cytometry. Top: representative data and gating strategy, with proliferation index (Prolif. index). Bottom: average data for $n=3-7$ pooled from at least 3 independent experiments. Statistics were performed using a 2-way ANOVA with Holm-Šídák post hoc test versus a $\triangle$ NGFR Treg control. * $P<0.05$; mean \pm SEM. (B-D) Irradiated NSG mice were injected with PBS $(n=3) ;$ HLA-A*02:01pos PBMCs alone $(n=$ 5); HLA-A*02:01 pos PBMCs and a 1:2 ratio of H1k2 hA2-CAR Tregs $(n=6)$; or mA2-CAR Tregs $(n=4)$. Data were pooled from 2 independent experiments. (B) Survival curve, log-rank (Mantel-Cox) test. (C) Human CD45+ engraftment upon experimental or humane end point (gating strategy in Supplemental Figure 7A). (D) Percent weight change at sacrifice relative to experiment start (day 49 or earlier). Statistical significance determined using a 1-way ANOVA and Holm-Šídák post hoc test; mean \pm SEM. ${ }^{*} P \leq 0.05,{ }^{* *} P \leq 0.01$.

of CARs, expression and specificity must be tested in $\mathrm{T}$ cells, and multiple activation markers should be screened to identify constructs with high, uniform expression without evidence for tonic signaling.

There are limited previous reports of humanized CARs (43-45). Sun et al. described a humanized antiHER2 CAR for use in breast cancer, but comparisons with the original murine construct were not reported (44). Johnson et al. humanized a CAR specific for the variant III mutation of the epidermal growth factor receptor (EGFRvIII) (43). Of 8 humanized constructs, only 2 remained EGFRvIII specific, and these had lower affinity than the original mouse CAR. We show here that sequence and structure-based approaches to 
A

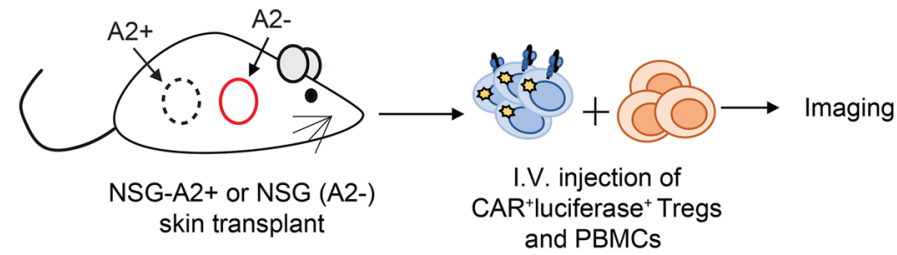

\section{B}

CAR-Treg Experiment

BMC<smiles>C1CCCCC1</smiles><smiles>C1CCC1</smiles>

\section{PBMC}

PBMC

and PBMCs
ํำ
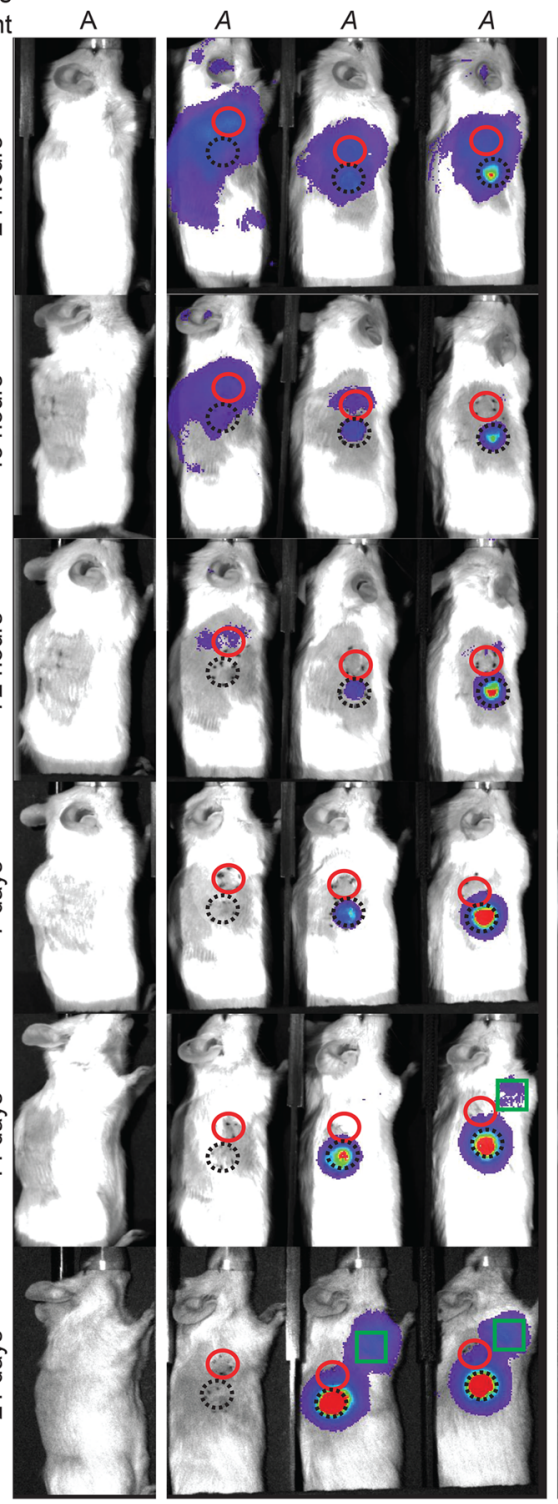

Radiance
Rec/cm $/ \mathrm{sr})$

Figure 6. Expression of $\mathrm{m} / \mathrm{hA2}$ CARs endows Tregs with rapid and persistent homing to HLA-A2:01+ skin allografts. Tregs were cotransduced with lentivirus encoding luciferase and either a control HER2CAR, mA2-CAR, or hA2-CAR (H1k2) and expanded for 7 days. Dual transduced cells were FACS sorted, expanded for 5 more days, then injected into NSC mice that had previously been transplanted with juxtaposed skin transplants from both NSG and NSG-HLA-A*02:01 transgenic mice. (A) Schematic representation of the experimental setup. (B) Representative luciferase imaging of skin grafts from 2 different experiments ( $A$ and $B$ ) at the indicated time points after Treg injection. Red solid circle denotes location of NSG skin graft; black dotted circle denotes location of A2-positive NSG skin graft; green square denotes location of draining lymph node with a visually detectable luciferase signal. Amount of luciferase radiance was quantified using the average amount of photons $/ \mathrm{s} / \mathrm{cm}^{2} /$ steradian and plotted as a ratio between the HLA-A*02:01NSG and NSG skin grafts (C) 72 hours after Treg injection or (D) over time. $n=6-7$ per group pooled from 3 independents experiments; mean \pm SEM. Repeated-measures ANOVA with Bonferroni's correction. ${ }^{*} P<0.05,{ }^{* *} P<0.01$.

\section{C}

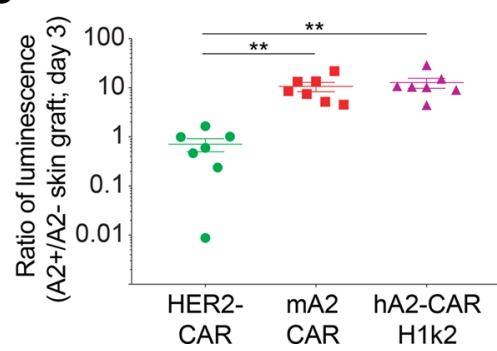

D

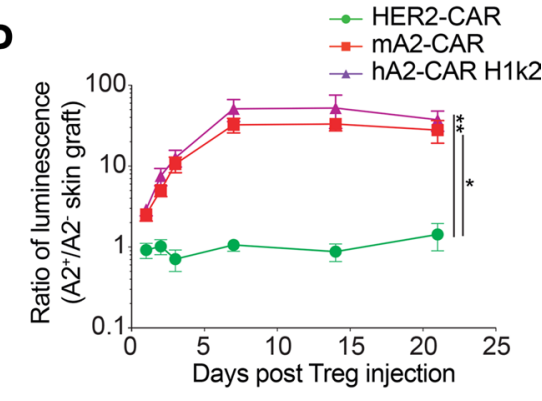

$\mathrm{NSG}$ A2+ 
A

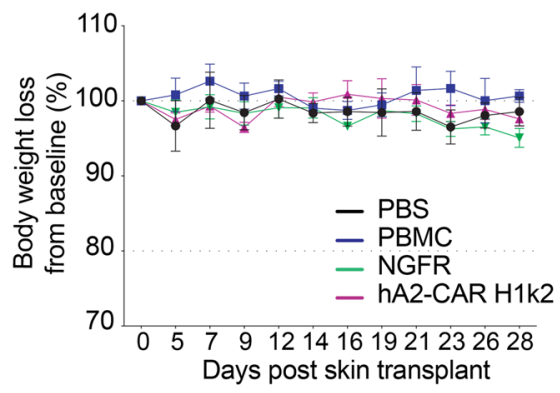

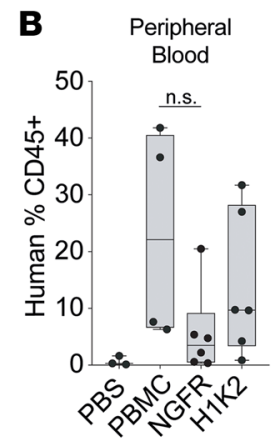

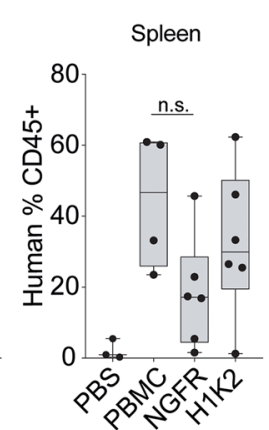

PBMC + H1k2 hA2-CAR Treg

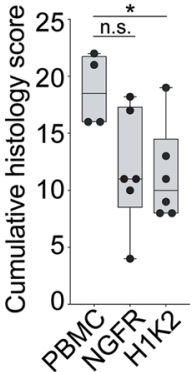

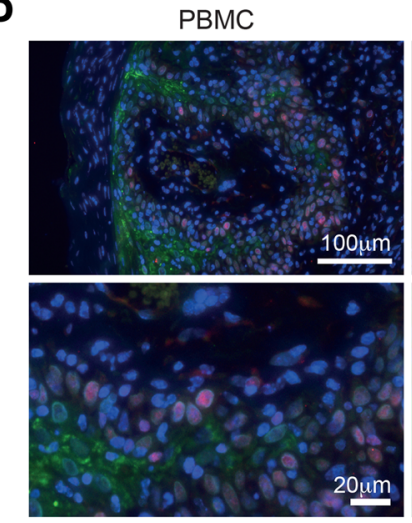

PBMC + NGFR Treg

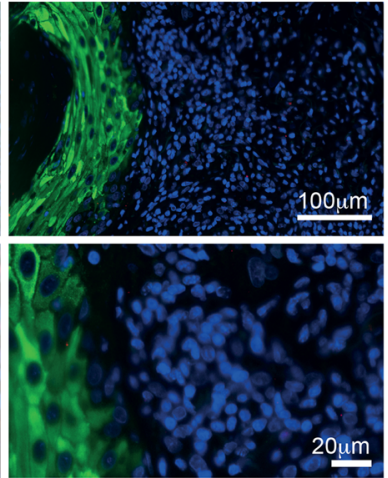

\section{DAPI
Ki-67
Involucrin
DAPI
Ki-67
Involucrin}
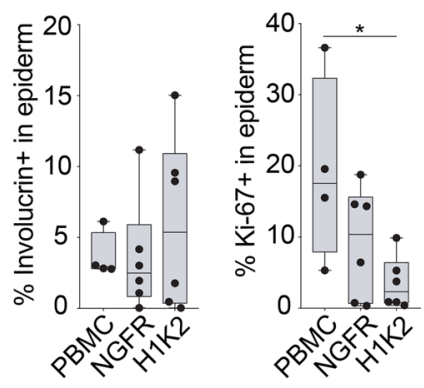

E
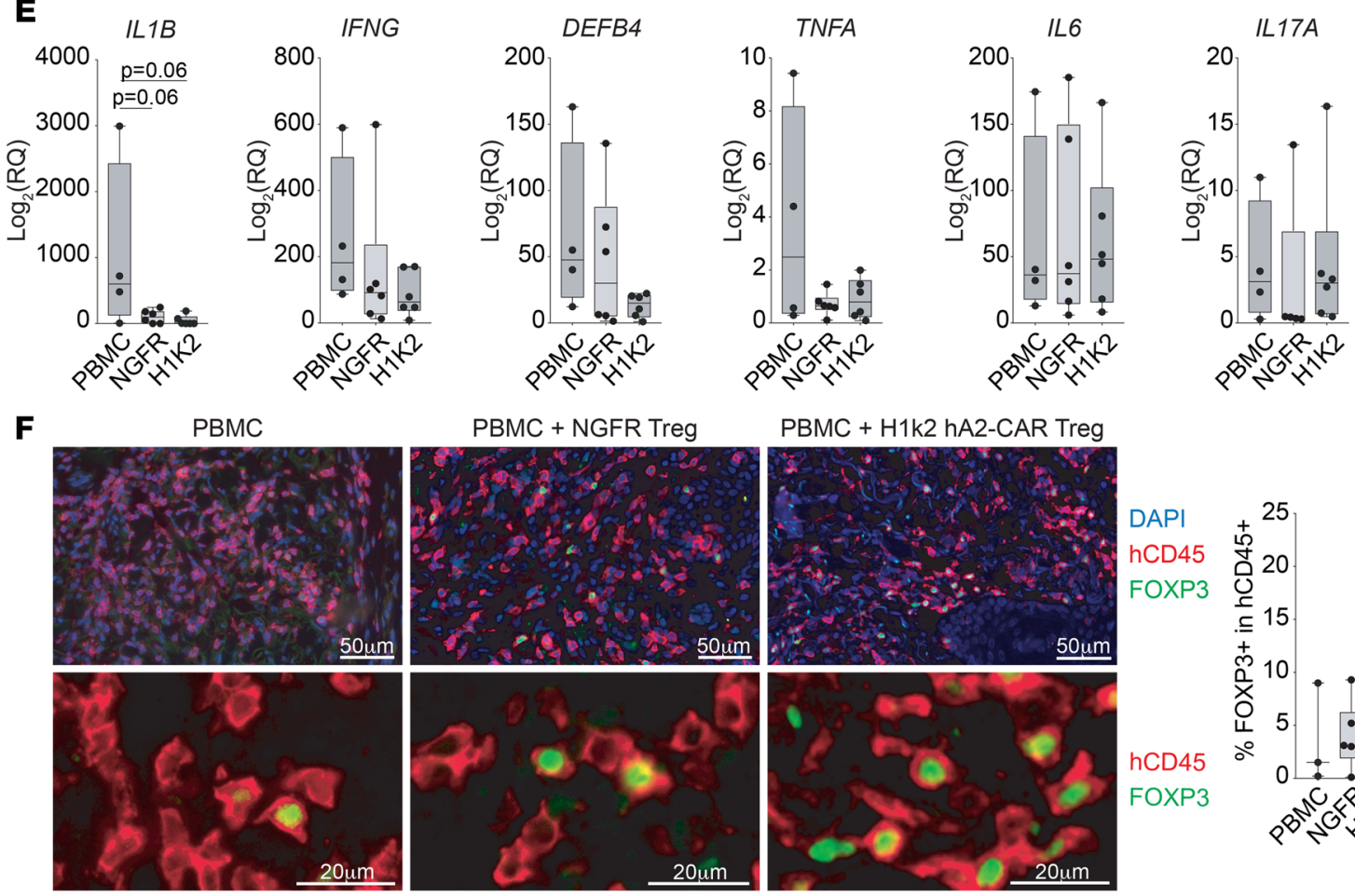

PBMC + H1k2 hA2-CAR Treg
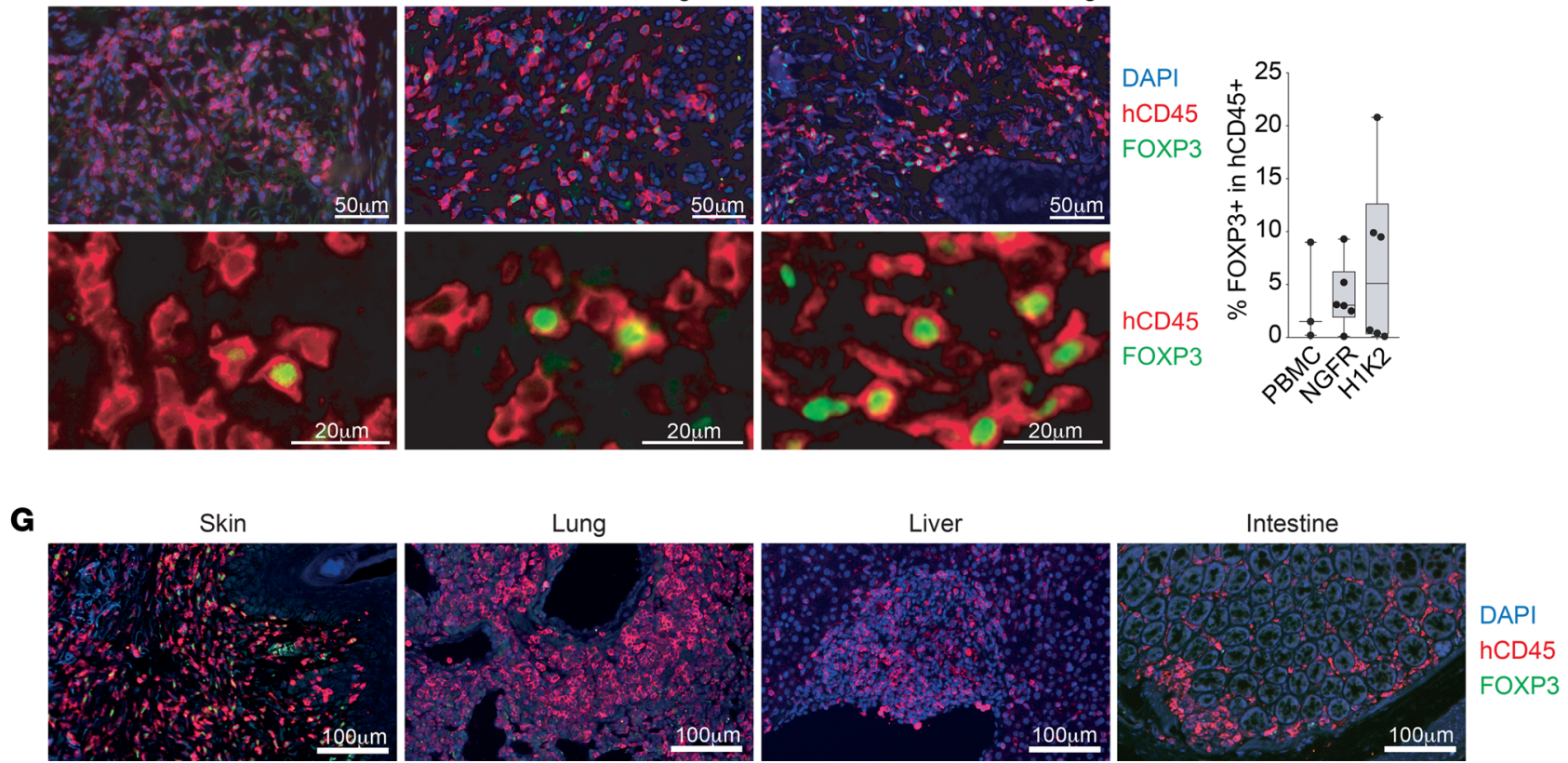
Figure 7. hA2-CAR Tregs diminish human skin allograft rejection. NSG mice were transplanted with HLA-A*02:01+ human skin and injected 6 weeks later with either PBS $(n=3)$, or HLA-A*02:01 1"eg PBMCs alone $(n=4)$ or with a 2:1 ratio of autologous H1k2 hA2-CAR Tregs (H1k2, $n=6)$ or $\triangle N G F R$ Tregs $(n=6)$. PBMCs/hA2-CAR Tregs were from 2 individual donors, tested in one experiment. Mice were monitored 3 times weekly and sacrificed 28 days after cell injection for mRNA and histology assessment. (A) Body weight was monitored 3 times weekly, and (B) the proportion of human CD45 cells in the blood (left) and spleen (right) was measured on day 28. (C) Cumulative histological score of transplanted skin sections as determined by H\&E staining. (D) Transplanted skin grafts were immunostained at experiment end point to quantify the amount of involucrin expression and proportion of $\mathrm{Ki}-67^{+}$cells in the epidermis. Scale bars: $100 \mu \mathrm{m}$ (top row), $20 \mu \mathrm{m}$ (bottom row). (E) mRNA expression of the indicated genes within transplanted skin sections was determined by qPCR. (F) Transplanted skin grafts harvested at the experiment endpoint were immunostained to quantify the proportion of $\mathrm{FOXP3}^{+}$cells within human $\mathrm{CD} 45^{+}$cells. Scale bars: $50 \mu \mathrm{m}$ (top row), $20 \mu \mathrm{m}$ (bottom row). (C) Transplanted skin grafts, intestine, lung, and liver sections were immunostained at the experiment end point to show the proportion of FOXP3 ${ }^{+}$cells within human $\mathrm{CD} 45^{+}$cells in each tissue. Scale bars: $100 \mu \mathrm{m}$. Each data point represents 1 mouse. Box-and-whisker plots show mean \pm range. Statistical significance determined by 1-way ANOVA comparing PBMCs with PBMCs + NGFR or H1k2. For immunofluorescence quantifications in $\mathbf{D}$ and $\mathbf{F}$, each data point represents the average cell number counted in $18-27$ fields of view from 1 section/mouse. ${ }^{*} P<0.05$.

antibody humanization can be used to generate humanized CARs (46-48), with empirical testing of multiple framework regions required to identify those with preserved expression and specificity without tonic signaling.

In the context of transplantation, knowledge of alloAg specificity is required to ensure specific targeting to allogeneic cells, tissues, and/or organs. The traditional way to measure $\mathrm{T}$ cell alloreactivity is imprecise and non-quantitative, as it involves functional MLRs with large banks of haplotyped PBMCs. We developed and validated a way to test the specificity of alloAg-directed CARs using commercially available reagents in common use to measure alloAg-specific Abs in serum. Surprisingly, we discovered that in comparison to the mA2-CAR, CAR humanization decreased cross-reactivity to several HLA-A allelic variants. All constructs also bound to $A * 69: 01$, and those containing $\mathrm{k} 2$ also to $A^{*} 68: 01$. This cross-reactivity is likely due to a shared eplet (138MT), an antibody-accessible polymorphic region of HLA defined by molecular modeling (49). Notably, the level of cross-reactivity toward $A * 25: 01$ or $A * 68: 01$ was not sufficient to stimulate $\mathrm{m} / \mathrm{hCAR}$ Treg activation, suggesting that a relatively high level of binding is required for a functional effect. This possibility is supported by data derived from analysis of $A^{*} 02: 01$, where the FlowPRT cell assay revealed a direct correlation between the relative binding of the CARs to $A^{*} 02: 01$ beads and induction of CD69. Nevertheless, lack of an effect on Treg activation marker expression does not exclude the possibility that there could be biological effects mediated by cross-reactive HLAs, since CAR-mediated T cell activation depends on both scFv affinity and avidity/Ag density, parameters that may not be fully recapitulated in this in vitro system (50).

The optimal CAR affinity for activation of Treg suppression is unknown. In terms of TCR affinity, it is known that TCR affinities for the same peptide-MHC complex can vary up to 3500-fold in Tregs, but ultimately, affinity has no effect on Ag-driven suppressive function (51). However, work from the CAR oncology field has shown that the structure and composition of the immunological synapses derived from CAR-peptide versus TCR-MHC/peptide interactions are fundamentally different (52). We found that CARs with the lowest tetramer binding (percent and MFI) had high apparent constitutive activity in some cases $(\mathrm{H} 4 \mathrm{k} 4, \mathrm{H} 5 \mathrm{k} 4)$ but not others ( $\mathrm{H} 2 \mathrm{k} 2)$, with a positive correlation between Ag binding and activation. Overall, more studies are required to determine the optimal Treg-specific synapse properties.

The homing of Tregs to allografts is key for their ability to induce tolerance to the grafts (reviewed in ref. 37). Here we found that $\mathrm{m} / \mathrm{hA2}$-CAR expression endowed Tregs with the ability to rapidly and specifically traffic to A2-expressing allografts. After trafficking to the A2 $2^{+}$graft, $\mathrm{m} / \mathrm{hA2}$-CAR Tregs (or their progeny), but not polyclonal Tregs, remained for at least 21 days. Other groups have also observed that continual Ag exposure enables long-term CAR Treg persistence, for example with CD19 CAR cytotoxic T cells $(18,53)$ and murine CAR Treg models of colitis (6) and islet transplantation (54). The Ag-driven persistence of Tregs is also supported by our findings in xenogeneic GvHD, where poor A2-CAR Treg persistence is correlated with diminished A2-positive immune cell engraftment (9). Interestingly, polyclonal HER2 CAR Tregs also showed a directed pattern of trafficking toward the allografts but were equally distributed between the A2-positive and -negative grafts. These polyclonal Tregs may be migrating in response to the inflammatory signals emanating from postoperative skin, because in unmanipulated immunodeficient mice, human $\mathrm{T}$ cells typically traffic to the lung (55). We also detected CAR Tregs in the draining lymph nodes in some mice. Since a 2-step migratory process from graft to lymph node has previously been reported to be necessary for tolerance induction (56), a more detailed investigation of CAR Treg trafficking is warranted.

Infusion of autologous hA2-CAR Tregs inhibited human skin allograft rejection as judged by an improved pathological score, a lower proportion of proliferating keratinocytes, and a trend toward diminished involucrin destruction. Consistent with previous reports in this model $(10,11)$, we noted modest 
differences between hA2-CAR Tregs and polyclonal ( $\triangle$ NGFR) Tregs. A consideration is that we used a relatively high Treg/PBMC ratio of 1:2. For comparison, Boardman et al. and Noyan. et al, who used lower A2-CAR Treg/PBMC ratios (1:5 and 1:7.5 ratios, respectively) (10, 11), also did not quantify significant differences between polyclonal and A2-CAR Tregs in terms of skin rejection. It is possible that in this model the intrinsic alloreactivity of polycloncal Tregs may be sufficient for a therapeutic effect even at relatively low Treg/PBMC ratios.

Importantly, A2-CAR Tregs did not make rejection worse in any reported studies (9-11), arguing against the possibility that after in vivo injection the cells acquired cytotoxic function via either Treg instability or outgrowth of contaminating conventional CAR $\mathrm{T}$ cells. To mitigate the risk of manufacturing CAR Tregs that were contaminated with low proportions of conventional $\mathrm{T}$ cells, for all in vivo studies we used naive $\mathrm{CD} 45 \mathrm{RA}^{+} \mathrm{CD} 25^{\text {hi }}$ Tregs as our starting population, since, in comparison to the CD45RA ${ }^{-}$memory Treg fraction, after expansion these cells maintain a more consistent Treg phenotype $(57,58)$. Nevertheless, the long-terms effects of repeated CAR stimulation on Tregs is unknown. Future work in immunocompetent mouse models will be needed to address this question, as well as the question of how these cells might be affected by standard immunosuppression regimens that would be used in transplant patients.

Collectively, these data provide a simple platform for CAR humanization and highlight the critical importance of testing several CARs in multiple assays to define optimal constructs for use in Tregs. Specifically, expressing/binding data in 293T cells were not consistently predictive of the construct properties in Tregs, as evidenced by the low expression of $\mathrm{H} 2 \mathrm{k} 2$ and bimodal expression patterns of the H4k4 and H5k4 variants. Furthermore, deficits in hA2-CAR Treg activation capacity were only revealed by testing multiple activation parameters. Consistent with the concept that Tregs require a strong Ag receptor signal, there was a direct correlation between strength of HLA binding and expression of activation and Treg functional markers. Evidence that hA2-CAR-engineered alloAg-specific Tregs persisted at the allograft, migrated to draining lymph nodes, and suppressed rejection sets the stage for testing their ability to regulate allograft rejection in humans.

\section{Methods}

Generation of humanized HLA-A*02-specific CARs. The humanized genes were codon optimized using the codon optimizer from the Thermo Fisher Scientific/Invitrogen GeneArt Gene Synthesis service, using the settings for Homo sapiens. gBlocks Gene Fragments were from Integrated DNA Technologies, such that the $5^{\prime}$ region of the CAR contained a Kozak sequence and a 36-nucleotide overlap with a pcDNA3 plasmid. The 3' end contained a BamHI site and an overlap with a CD8 hinge sequence to facilitate Gibson assembly into the plasmid in frame with the CD8 hinge and CAR intracellular signaling domains.

The $\mathrm{scFv}$ variants were fused to a stalk region from human $\mathrm{CD} 8 \alpha$; the transmembrane and intracellular domains were from human $\mathrm{CD} 28$ and human $\mathrm{CD} 3 \zeta$ as described previously $(9,59)$. The resulting cDNAs were cloned into a lentiviral vector encoding $\triangle$ NGFR as a marker. Surface expression was determined by flow cytometry with transiently transfected HEK293T cells (jetPRIME, Polyplus Transfection). Viral particles were produced as described previously (60).

Generation of HLA-expressing K562 cell lines. CD64-expressing K562 cells (K562.64) were a gift from James Riley, University of Pennsylvania, Philadelphia, Pennsylvania, USA. cDNA for HLA-A*02:01 and -A*24:02 was isolated from mRNA of PBMCs from a donor homozygous for $A^{*} 02: 01$ or $A^{*} 24: 02$, respectively, on the HLA-A locus using the following primer sequences: 5'-TTTTCTAGACGCGTGCCACCATGGCCGTCATGGCGCC-3' (forward) and 5'-AAGTCGACGCTAGCTCACACTTTACAAGCTGTGAGAGACA-3' (reverse). The resulting sequence was confirmed by Sanger sequencing, aligned to the expected sequences from the Immuno Polymorphism Database (IPD) and international IMGT/HLA Database (61), and transduced into K562 cells, respectively, using a lentiviral expression vector. To generate A25-, A68-, or A69-K562 cells, HLA sequences for $A * 25: 01, A * 68: 01$, and $A * 69: 01$ were scraped from the IPD and IMGT/HLA Database (61), codon optimized using the codon optimizer tool (set to Homo sapiens) from Thermo Fisher Scientific/Invitrogen GeneArt Gene Synthesis service, then cloned into a lentiviral expression vector and transduced into K562 cells. The resulting K562 cell lines were then sorted on a FACSAria II (BD Biosciences) using anti-HLA-ABC (Thermo Fisher Scientific, 12-9983-41) to ensure equivalent surface expression of the transduced HLA, and anti-HLA-A2 (BB7.2) (Thermo Fisher Scientific, 17-9876-42) to ensure purity.

Treg sorting, transduction, and expansion. $\mathrm{CD}^{+} \mathrm{T}$ cells were isolated from HLA-A2- donors via RosetteSep (STEMCELL Technologies, 15062) and enriched for CD25+ cells (Miltenyi Biotec, 130-092-983) prior to sorting live $\mathrm{CD} 4^{+} \mathrm{CD} 25^{\mathrm{hi}} \mathrm{CD} 127^{\text {lo }}$ Tregs (for in vitro and luciferase experiments) or $\mathrm{CD} 4^{+} \mathrm{CD} 127^{\text {lo }} \mathrm{C}$ - 
D25 ${ }^{\text {hi }}$ CD45RA ${ }^{+}$Tregs (for in vivo transplant experiments) using a MoFlo Astrios (Beckman Coulter) or FACSAria II (BD Biosciences), respectively. Sorted Tregs were stimulated with L cells and $\alpha \mathrm{CD} 3 \mathrm{mAb}$ (OKT3, UBC AbLab; $100 \mathrm{ng} / \mathrm{ml}$ ) in ImmunoCult-XF T cell expansion media (STEMCELL Technologies, 10981) with $1000 \mathrm{U} / \mathrm{ml} \mathrm{IL-2} \mathrm{(Proleukin)} \mathrm{as} \mathrm{described} \mathrm{previously} \mathrm{(9).} \mathrm{One} \mathrm{day} \mathrm{later,} \mathrm{cells} \mathrm{were} \mathrm{transduc-}$ ed with lentivirus at a multiplicity of infection of 10 virus particles: 1 cell. As a positive control, Tregs were transduced with the mA2-CAR construct as described (9). To generate polyclonal Treg controls, cells were transduced with a vector encoding either a HER2-CAR and the $\triangle$ NGFR marker, or only $\triangle$ NGFR. The latter was used for in vivo skin transplant experiments to avoid HER2-mediated activation via HER2 expressed by human skin. On day $7, \Delta \mathrm{NGFR}^{+}$cells were purified with magnetic selection (Miltenyi Biotec, 130-091-330), then used in assays or restimulated with $L$ cells as above and expanded for 5 days for in vivo experiments. To test the effects of HLA-A2-mediated stimulation, Tregs were cultured with limiting IL-2 $(100 \mathrm{U} / \mathrm{mL})$ for 24 hours, then recounted and cocultured with irradiated anti-CD3/anti-CD28-loaded CD64-expressing K562 cells, or with HLA-A*02:01-, HLA-A*24:02-, HLA-A*25:01, HLA-A*68:01-, or HLA-A*69:01-expressing K562 cells, at a 1:2 (K562/T cell) ratio for 24 hours.

Flow cytometry. For phenotypic analysis, cells were stained with fixable viability dye (FVD, Thermo Fisher Scientific, 65-0865-14; BioLegend, 423102) and for surface markers before fixation and permeabilization using an eBioscience FOXP3/Transcription Factor Staining Buffer Set (Thermo Fisher Scientific, 00-5523-00) and staining for intracellular proteins. Samples were read on a CytoFLEX (Beckman Coulter) and results analyzed using FlowJo Software version 9.9.4 and 10.3 (Tree Star).

Surface staining was performed for NGFR (Miltenyi Biotec, 130-091-885), CD3 (BD Biosciences, 564465), CD4 (BioLegend, 317410), CD25 (Miltenyi Biotec, 130-091-024), LAP (Thermo Fisher Scientific, 25-9829-42), CD69 (BioLegend, 310946), CD71 (BD Biosciences, 563768), and CD127 (Thermo Fisher Scientific, 48-1278-42). Tetramer staining was performed with HLA-A*02:01 monomers provided by the NIH Tetramer Core Facility (Emory University, Atlanta, Georgia, USA) made into tetramers with streptavidin-allophycocyanin (ProZyme, PJ27S). Intracellular staining was performed for CTLA-4 (BioLegend, 369606).

For in vivo experiments, $50 \mu 1$ blood was collected weekly and at end points. Ammonium chloride was used for red blood cell lysis. Cells were resuspended in PBS with anti-mouse CD16/32 (Thermo Fisher Scientific, 14-0161-82) and stained for extracellular markers using FVD, anti-mouse CD45 (Thermo Fisher Scientific, 25-0451-82), and anti-human CD45 (BD Biosciences, 560777), CD4 (BioLegend, 300554, 317434), CD8 (Thermo Fisher Scientific, 48-0087-42), anti-human CD271 (NGFR; BD Biosciences, 557196), HLA-A2 (BD Biosciences, 551285). Intracellular staining for FOXP3 (Thermo Fisher Scientific, 12-4777-42) was done with the eBioscience FOXP3/Transcription Factor Staining Buffer Set (Thermo Fisher Scientific, 00-5523-00). 10,000 counting beads were added to every sample (Thermo Fisher Scientific, 01-1234-42). The gating strategies for the xenogeneic GvHD, luciferase, and skin transplant experiments are illustrated in Supplemental Figure 7A, Supplemental Figure 8B, and Supplemental Figure 9A, respectively.

$H L A$ allele cross-reactivity assay. $0.025 \times 10^{6} \mathrm{CAR}$ Tregs (prepared as above, after 7 days of culture) were incubated with individual FlowPRA Single Antigen bead panels (FL1HD01, FL1HD02, FL1HD03, FL1HD04, FL1HD06, and FL1HD08, One Lambda) and FVD for 30 minutes at room temperature. Samples were washed, fixed with $0.5 \%$ formaldehyde, and analyzed via flow cytometry. Two hundred negative control beads were acquired per sample.

For analysis, single Ag beads were gated after exclusion of dead cells and doublets, and the number of beads for each HLA Ag was quantified within each distinct PE intensity peak. To account for small variations in the absolute number of negative control beads collected in each sample, data were normalized by multiplying the number of beads of interest in each HLA peak by 200, divided by the number of negative beads in the sample. Then, relative binding of A2-CAR Tregs compared with $\triangle$ NGFR CAR was obtained by dividing the average number of beads in the $\triangle$ NGFR specimen for a specific HLA minus the normalized number of beads in the A2-CAR Treg specimen for that same HLA by the average number of beads in the $\triangle$ NGFR specimen, times 100 .

Suppression of MLRs. Adherent cells from PBMCs from HLA-A2 ${ }^{+}$healthy donors were differentiated into monocyte-derived dendritic cells as described previously (62). For MLRs, HLA-A2- PBMC responder cells were labeled with cell proliferation dye eF450 (Thermo Fisher Scientific, 65-0842-85), then plated with $5 \times 10^{4} \mathrm{HLA}-\mathrm{A} 2^{+}$monocyte-derived dendritic cells and increasing ratios of expanded $\triangle$ NGFR- or hA2-CAR-expressing Tregs labeled with cell proliferation dye e670 (Thermo Fisher 
Scientific, 65-0840-90). After 6 days, division of HLA-A2- CD4 ${ }^{+}$responder T cells was measured by flow cytometry. Percent suppression was calculated based on the proliferation index of a given cell combination and ratio versus the positive control (HLA-A2 ${ }^{+}$monocyte-derived dendritic cells with HLA-A2- $\mathrm{CD}^{+}{ }^{+}$responder $\mathrm{T}$ cells only), as described previously (63). Data were normalized by first calculating percent suppression as follows:

$\%$ suppression $=100-100 \times \frac{\text { proliferation index }(\text { sample })}{\text { proliferation index }(D C+\text { responder control })}$

\section{Equation 1}

then normalizing the resulting values from $0 \%-100 \%$, according to the formula for each independent experiment:

normalized $\%$ suppression $=100 \times \frac{\% \text { suppression }(\text { sample })}{\% \text { suppression }(D C+\text { responder control })}$

Equation 2

Xenogeneic graft-versus-host disease. Eight- to 12-week-old female NSG mice (The Jackson Laboratory; bred in house) received whole-body irradiation (150 cGy, Rad Source Technologies RS-2000 Pro Biological System) 1 day before injection of $8 \times 10^{6} \mathrm{HLA}-\mathrm{A} 2^{+} \mathrm{PBMCs}$ with or without $4 \times 10^{6} \mathrm{hA} 2$-CAR Tregs intravenously into the tail vein. Saline-injected mice served as controls. m/hA2-CAR Tregs were generated from 4 healthy donors. GvHD was scored based on weight, fur texture, posture, activity level, and skin integrity, with $0-2$ points per category as described previously $(64,65)$. Peripheral blood from the saphenous vein was centrifuged; then erythrocytes were lysed, and leukocytes were measured by flow cytometry.

Luciferase. To evaluate Treg homing toward HLA-A2-expressing mouse skin grafts in vivo, sorted Tregs $\left(\mathrm{CD} 4{ }^{+} \mathrm{CD} 25^{\mathrm{hi}} \mathrm{CD} 127^{\mathrm{lo}}\right)$ were stimulated with $\mathrm{L}$ cells as described above. The next day, cells were transduced with HER2-CAR, mA2-CAR, or H1k2 hA2-CAR lentivirus at an MOI of 10 and 8 hours later with luciferase-GFP lentivirus at an MOI of 5. The lentiviral plasmid encoding a beetle luciferase-GFP fusion protein (pELNS.CBG-T2A-GFP [CBR]) was provided by David Barrett (Children's Hospital of Philadelphia, Philadelphia, Pennsylvania, USA) (39). After 7 days of culture, double-transduced $\mathrm{GFP}^{+} \Delta \mathrm{NGFR}^{+}$Tregs expressing the CAR and luciferase were sorted before restimulation with $\mathrm{L}$ cells as described above. On day 12 of the culture, $1 \times 10^{6}$ to $3 \times 10^{6}$ luciferase-CAR Tregs and $6 \times 10^{6}$ human allogeneic HLA-A2- PBMCs were injected intravenously into skin-transplanted NSG mice. For bioluminescence imaging, D-luciferin potassium salt (150 mg/kg, GoldBio) was injected intraperitoneally immediately before anesthesia with isoflurane, and images were acquired within 15-20 minutes on an Ami-X (Spectral Instruments Imaging). Data were analyzed with AmiView software (Spectral Instruments Imaging, version 1.7.06), and the luminescent signal was quantified as the ratio of photons $/ \mathrm{s} / \mathrm{cm}^{2} /$ steradian in the HLA-A2 ${ }^{+}$over the HLA-A2 ${ }^{-}$skin graft, or the ratio of the relevant lymph node area over the HLA-A2- skin graft. At experimental end points, skin-draining axillar lymph nodes and spleen were harvested, placed on a $70-\mu \mathrm{m}$ cell strainer (BD Falcon), then fragmented and filtered by using the plunger of a 1-cc syringe. Cells were then stained for flow cytometry.

Skin transplantation. To evaluate A2-CAR Treg homing and capacity to inhibit skin rejection, 8- to 12-week-old female NSG mice (The Jackson Laboratory, bred in house) were transplanted with skin from transgenic HLA-A2 ${ }^{+}$NSG mice (The Jackson Laboratory, bred in house), NSG skin (HLA-A2 negative), or human HLA-A2 ${ }^{+}$skin. For mouse skin transplants, skin was cut into circular pieces utilizing an 8-mm biopsy punch, placed onto fresh plates with PBS, and kept at $4-8^{\circ} \mathrm{C}$ until transplanted ( $\sim 1-4$ hours). HLA-A2 expression of human skin was assessed by flow cytometry and qPCR. Split-thickness skin explants were generated by trimming fat and rinsing with sterile PBS, then cut into $1-\mathrm{cm}^{2}$ pieces, placed onto fresh plates with PBS and kept at low temperature $\left(4-8^{\circ} \mathrm{C}\right)$ until transplanted $(\sim 1-4$ hours). For both mouse and human skin transplants, previously shaved mice were anesthetized, dorsal skin was cut near the shoulder, and mouse skin of similar size was removed, then grafts were placed on the exposed area and stabilized with steri-strips (3M, Nexcare). Grafts were covered with a Vaseline gauze and wrapped with a 2-cm-wide CoFlex bandage (3M, Nexcare) to secure the graft for up to 14 days prior to cell injection.

Histology. Human skin grafts and surrounding mouse skin were harvested 28 days after cell injection, fixed overnight at $4^{\circ} \mathrm{C}$ in $10 \%$ formalin $(1: 10 \mathrm{v} / \mathrm{v}$ ratio of tissue to formalin), and stored in $70 \%$ ethanol before paraffin embedding. Paraffin sections ( $5-\mu \mathrm{m}$ thickness) and H\&E staining were prepared by BCCHR Histology Services. For immunostaining, sections were deparaffinized and rehydrated using a series of 
xylene washes $(\times 3)$, graded alcohol solutions $(2 \times 100 \%$ ethanol, $1 \times 95 \%$ ethanol, and $1 \times 70 \%$ ethanol), and $1 \times$ PBS. Heat-induced epitope retrieval (HIER) was performed on slides using a microwave to reach $93-95^{\circ} \mathrm{C}$ ( 5 minutes high power followed by 20 minutes low power) in $10 \mathrm{mM}$ sodium citrate buffer $(0.5 \%$ Tween-20, pH 6.0). Following HIER, slides were washed using running tap water, deionized water, and PBS. Sections were incubated with DProtein Block, Serum-Free (Dako, X0909) to limit nonspecific antibody staining. Sections were then incubated at $4^{\circ} \mathrm{C}$ overnight with the following primary antibodies: FOXP3 (Invitrogen, clone PCH101, 14-4776-82), CD45 (eBioscience, clone H130, 17-0459), Ki-67 (eBioscience, clone 20Raji, 17-5699), involucrin (Abcam, ab53112). The following day, sections were gently rinsed with PBS several times, then stained for 1 hour at room temperature with the following secondary antibodies: donkey anti-rat-Alexa Fluor 488 (Life Tech, A11006), goat anti-mouse-APC (Invitrogen, 1834696), donkey anti-rabbit-Alexa Fluor 488488 (Life Tech, A21206). Finally, sections were counterstained with DAPI to identify cell nuclei and mounted using VECTASHIELD Mounting Medium with DAPI (Vector Laboratories, H-1200). All antibodies were diluted in Antibody Diluent (Dako, S3022). Images were captured using an Olympus BX61 Fluorescence and Bright Field Automated Upright Microscope with QImaging Retiga Exi camera and Olympus DP71 color camera. Quantitative analysis of fluorescence images was performed using Fiji with Olympus viewer plug-in $(66,67)$. Immunofluorescence images were quantified by counting the number of indicated cells in 18-27 different fields of view from one section.

H\&E-stained slides were evaluated in a blinded manner by a clinical pathologist using a scoring system defined by 8 factors, each graded from 0 to 3-4; Lerner grade $(0,1$ : focal or diffuse vacuolar degeneration, 2: dyskeratosis, 3: clefts in basal or superficial layers, 4: frank loss of epidermis), spongiosis ( 0 , 1: basal layer only, 2 : up to halfway, 3 : full thickness), necrotic keratinocytes $(0,1:$ rare $[1 / \mathrm{hpf}], 2$ : occasional [2-3/hpf], 3: many [ $>4 / \mathrm{hpf}])$, necrotic keratinocyte location $(0,1$ : basal only, 2 : up to upper half, 3: full thickness), satellitosis ( $0,1: 1$ only, $2: 2-3 / \mathrm{hpf}, 3: \geq 4 / \mathrm{hpf}$, exocytosis $(0,1$ : focal, $2:<50 \%$ biopsy, 3: $>50 \%$ biopsy), adnexal involvement $(0,1:$ minor involvement of any adnexa: 2 : marked involvement of $<50 \%$ adnexa, 3 : marked involvement of $>50 \%$ adnexa), and lymphoid cuffs in dermis ( 0,1 : slight, 2 : abundant, 3: band like) (68-70).

$q P C R$. RNA was harvested from human skin samples according to the manufacturer's instructions (RNeasy Plus Mini Kit; QIAGEN) and converted to cDNA. qPCR was performed using SYBR green (BioRad) and primers for $I L 17, I L 6, I L 1 B, D E F B 4, I F N g, T N F a$, and $18 S$ ribosomal RNA (Supplemental Table 2). Melt curve and SYBR green emission data were collected. Relative concentrations were calculated using a standard curve, and values were normalized to amplification products of $18 S$ ribosomal RNA. $\log _{2}$ (RQ) values for each sample were obtained using the double delta $\mathrm{Ct}(\Delta \Delta \mathrm{Ct})$ method (71). Each sample's $\Delta \mathrm{Ct}$ value was obtained by calculating averaged Ct (gene of interest) - Ct (housekeeping gene). To obtain $\Delta \Delta \mathrm{Ct}$, the $\Delta \mathrm{Ct}$ of the control sample was subtracted from the $\Delta \mathrm{Ct}$ of the treated sample. Fold gene expression was then calculated by $2^{-(\Delta \Delta C t)}$.

Statistics. All statistics were done using Prism 7.0b. IBM*SPSS Statistics version 24.0.0.0 was used for Figure 6D. For all studies, normality was assumed. Corrections for multiple comparisons were made as described in each figure.

Study approval. For human PBMCs used for in vitro and skin homing in vivo experiments, healthy volunteers gave written informed consent according to protocols approved by the University of British Columbia Clinical Research Ethics Board (UBC-CREB) and Canadian Blood Services. For in vivo xeno-GvHD and human skin transplant studies, commercial leukapheresis blood products were purchased from STEMCELL Technologies excluding all variants of the following HLA alleles: $A^{*} 02, A^{*} 68, A^{*} 69$. Samples of human skin discarded from plastic surgery were obtained from the Harvard Skin Disease Resource Center (Brigham \& Women's Hospital, Harvard Medical School), Skinworks (Vancouver), or the Cambie Surgery Clinic (Vancouver) according to UBC-CREB-approved protocols. Animal protocols were approved by the UBC Animal Care Committee.

\section{Author contributions}

NAJD and CL conceived, designed and conducted experiments, analyzed data, and wrote the manuscript. REH conceived, designed, and conducted experiments, analyzed data, and critically reviewed the manuscript. PB performed in silico antibody humanization and critically reviewed the manuscript. VF, EM, QH, MS, and JG conducted experiments, analyzed data, and critically reviewed the manuscript. PCO provided cloning guidance and critically reviewed the manuscript. JWB designed and performed the histology scor- 
ing for the skin transplant experiment. MM conceived, designed, and conducted experiments, analyzed data, and critically reviewed the manuscript. MKL secured funding, conceived and designed experiments, provided overall direction and interpretation, and wrote the manuscript.

\section{Acknowledgments}

The authors thank the Human Skin Disease Resource Center at Harvard University, as well as Nicholas Carr, Nancy Van Laeken, David Granville, and Valerio Lusso (all UBC) for assistance with human skin retrieval. We thank David Barrett (Children's Hospital of Philadelphia) for the luciferase-GFP fusion construct, Canadian Blood Services for providing blood samples, Lisa Xu for flow cytometry support, and Michelle Le for help with cloning the hA2-CAR constructs. This work was supported by grants from the Canadian Institutes of Health Research (CIHR; FDN-154304) and TxCell (to MKL). MKL and REH receive salary awards from the BC Children's Hospital Research Institute. NAJD is supported by a CIHR doctoral award; CL is supported by a CIHR and a KRESCENT fellowship award. VCWF receives an American Society for Transplantation Student Internship Research Program salary award.

Address correspondence to: Megan K. Levings, BC Children's Hospital Research Institute, A4-186, 950 West 28th Ave., Vancouver, British Columbia. Canada, V5Z 4H4. Phone; 604.875.2000 ext 4686; Email: mlevings@bcchr.ca.

1. Gliwiński M, Iwaszkiewicz-Grześ D, Trzonkowski P. Cell-based therapies with T regulatory cells. BioDrugs. 2017;31(4):335-347.

2. Dawson NAJ, Vent-Schmidt J, Levings MK. Engineered tolerance: tailoring development, function, and antigen-specificity of regulatory T cells. Front Immunol. 2017;8:1460.

3. Putnam AL, et al. Clinical grade manufacturing of human alloantigen-reactive regulatory T cells for use in transplantation. $A m$ J Transplant. 2013;13(11):3010-3020.

4. Tang Q, Lee K. Regulatory T-cell therapy for transplantation: how many cells do we need? Curr Opin Organ Transplant. 2012;17(4):349-354

5. Elinav E, Adam N, Waks T, Eshhar Z. Amelioration of colitis by genetically engineered murine regulatory T cells redirected by antigen-specific chimeric receptor. Gastroenterology. 2009;136(5):1721-1731.

6. Elinav E, Waks T, Eshhar Z. Redirection of regulatory T cells with predetermined specificity for the treatment of experimental colitis in mice. Gastroenterology. 2008;134(7):2014-2024.

7. Fransson M, et al. CAR/FoxP3-engineered T regulatory cells target the CNS and suppress EAE upon intranasal delivery. $J$ Neuroinflammation. 2012;9:112.

8. Yoon J, Schmidt A, Zhang AH, Königs C, Kim YC, Scott DW. FVIII-specific human chimeric antigen receptor T-regulatory cells suppress T- and B-cell responses to FVIII. Blood. 2017;129(2):238-245.

9. MacDonald KG, et al. Alloantigen-specific regulatory T cells generated with a chimeric antigen receptor. J Clin Invest. 2016;126(4):1413-1424.

10. Boardman DA, et al. Expression of a chimeric antigen receptor specific for donor HLA class I enhances the potency of human regulatory T cells in preventing human skin transplant rejection. Am J Transplant. 2017;17(4):931-943.

11. Noyan F, et al. Prevention of allograft rejection by use of regulatory T cells with an MHC-specific chimeric antigen receptor. Am J Transplant. 2017;17(4):917-930.

12. Wu Y, Jiang S, Ying T. From therapeutic antibodies to chimeric antigen receptors (CARs): making better CARs based on antigen-binding domain. Expert Opin Biol Ther. 2016;16(12):1469-1478.

13. Lee DW, et al. T cells expressing CD19 chimeric antigen receptors for acute lymphoblastic leukaemia in children and young adults: a phase 1 dose-escalation trial. Lancet. 2015;385(9967):517-528.

14. Maude SL, et al. Chimeric antigen receptor T cells for sustained remissions in leukemia. N Engl J Med. 2014;371(16):1507-1517.

15. Grupp SA, et al. Chimeric antigen receptor-modified T cells for acute lymphoid leukemia. N Engl J Med. 2013;368(16):1509-1518.

16. Porter DL, Levine BL, Kalos M, Bagg A, June CH. Chimeric antigen receptor-modified T cells in chronic lymphoid leukemia. N Engl J Med. 2011;365(8):725-733.

17. Hege KM, et al. Safety, tumor trafficking and immunogenicity of chimeric antigen receptor (CAR)-T cells specific for TAG-72 in colorectal cancer. J Immunother Cancer. 2017;5:22.

18. Lamers $\mathrm{CH}$, et al. Parallel detection of transduced T lymphocytes after immunogene therapy of renal cell cancer by flow cytometry and real-time polymerase chain reaction: implications for loss of transgene expression. Hum Gene Ther. 2005;16(12):1452-1462.

19. Kershaw $\mathrm{MH}$, et al. A phase I study on adoptive immunotherapy using gene-modified T cells for ovarian cancer. Clin Cancer Res. 2006;12(20 Pt 1):6106-6115.

20. Turtle CJ, et al. CD19 CAR-T cells of defined CD4+:CD8+ composition in adult B cell ALL patients. J Clin Invest. 2016;126(6):2123-2138.

21. Maude SL, et al. Efficacy of humanized CD19-targeted chimeric antigen receptor (CAR)-modified t cells in children and young adults with relapsed/refractory acute lymphoblastic leukemia. Blood. 2016;128(22):217.

22. Maus MV, et al. T cells expressing chimeric antigen receptors can cause anaphylaxis in humans. Cancer Immunol Res. 2013;1:26-31.

23. Lamers $\mathrm{CH}$, et al. Immune responses to transgene and retroviral vector in patients treated with ex vivo-engineered $\mathrm{T}$ cells. Blood. 2011;117(1):72-82.

24. Hwang WY, Foote J. Immunogenicity of engineered antibodies. Methods. 2005;36(1):3-10. 
25. Hansel TT, Kropshofer H, Singer T, Mitchell JA, George AJ. The safety and side effects of monoclonal antibodies. Nat Rev Drug Discov. 2010;9(4):325-338.

26. Jones PT, Dear PH, Foote J, Neuberger MS, Winter G. Replacing the complementarity-determining regions in a human antibody with those from a mouse. Nature. 1986;321(6069):522-525.

27. Verhoeyen M, Milstein C, Winter G. Reshaping human antibodies: grafting an antilysozyme activity. Science. 1988;239(4847):1534-1536.

28. Queen C, et al. A humanized antibody that binds to the interleukin 2 receptor. Proc Natl Acad Sci USA. 1989;86(24):10029-10033.

29. Kashmiri SV, De Pascalis R, Gonzales NR, Schlom J. SDR grafting — a new approach to antibody humanization. Methods. 2005;36(1):25-34

30. Suzuki M, Kato C, Kato A. Therapeutic antibodies: their mechanisms of action and the pathological findings they induce in toxicity studies. J Toxicol Pathol. 2015;28(3):133-139.

31. Riechmann L, Clark M, Waldmann H, Winter G. Reshaping human antibodies for therapy. Nature. 1988;332(6162):323-327.

32. Meyer D, C Aguiar VR, Bitarello BD, C Brandt DY, Nunes K. A genomic perspective on HLA evolution. Immunogenetics. 2018;70(1):5-27.

33. Kabat EA, Wu TT, Perry HM, Gottseman KS, Foeller C. Sequences of proteins of immunological interest. Bethesda, Maryland, USA: US Dept. of Health Human Services, Public Health Service; 1991. NIH 91-3242.

34. Chothia C, Lesk AM. Canonical structures for the hypervariable regions of immunoglobulins. J Mol Biol. 1987;196(4):901-917.

35. El-Awar N, Lee JH, Tarsitani C, Terasaki PI. HLA class I epitopes: recognition of binding sites by mAbs or eluted alloantibody confirmed with single recombinant antigens. Hum Immunol. 2007;68(3):170-180.

36. Hilton HG, Parham P. Direct binding to antigen-coated beads refines the specificity and cross-reactivity of four monoclonal antibodies that recognize polymorphic epitopes of HLA class I molecules. Tissue Antigens. 2013;81(4):212-220.

37. Lamarche C, Levings MK. Guiding regulatory T cells to the allograft. Curr Opin Organ Transplant. 2018;23(1):106-113.

38. Lam AJ, Hoeppli RE, Levings MK. Harnessing advances in T regulatory cell biology for cellular therapy in transplantation. Transplantation. 2017;101(10):2277-2287.

39. Barrett DM, et al. Relation of clinical culture method to T-cell memory status and efficacy in xenograft models of adoptive immunotherapy. Cytotherapy. 2014;16(5):619-630.

40. Cao J, et al. Potent anti-leukemia activities of humanized CD19-targeted Chimeric antigen receptor T (CAR-T) cells in patients with relapsed/refractory acute lymphoblastic leukemia. Am J Hematol. 2018;93(7):851-858.

41. Lamers $\mathrm{CH}$, et al. Treatment of metastatic renal cell carcinoma with autologous T-lymphocytes genetically retargeted against carbonic anhydrase IX: first clinical experience. J Clin Oncol. 2006;24(13):e20-e22.

42. Safdari Y, Farajnia S, Asgharzadeh M, Khalili M. Antibody humanization methods - a review and update. Biotechnol Genet Eng Rev. 2013;29:175-186.

43. Johnson LA, et al. Rational development and characterization of humanized anti-EGFR variant III chimeric antigen receptor T cells for glioblastoma. Sci Transl Med. 2015;7(275):275ra22.

44. Sun M, Shi H, Liu C, Liu J, Liu X, Sun Y. Construction and evaluation of a novel humanized HER2-specific chimeric receptor. Breast Cancer Res. 2014;16(3):R61.

45. Qian L, et al. The novel anti-CD19 chimeric antigen receptors with humanized scFv (single-chain variable fragment) trigger leukemia cell killing. Cell Immunol. 2016;304-305:49-54.

46. Teplyakov A, et al. Structural insights into humanization of anti-tissue factor antibody 10H10. MAbs. 2018;10(2):269-277.

47. Schwaigerlehner L, Pechlaner M, Mayrhofer P, Oostenbrink C, Kunert R. Lessons learned from merging wet lab experiments with molecular simulation to improve mAb humanization. Protein Eng Des Sel. 2018;31(7-8):257-265.

48. Choi Y, Hua C, Sentman CL, Ackerman ME, Bailey-Kellogg C. Antibody humanization by structure-based computational protein design. MAbs. 2015;7(6):1045-1057.

49. Zeevi A, Marrari M, Feingold B, Webber S, Duquesnoy RJ. Human leukocyte antigen epitope analysis to assess complement- and non-complement-binding donor-specific antibody repertoire in a pediatric heart transplant recipient. Hum Immunol. 2012;73(1):48-51

50. Ramos CA, Dotti G. Chimeric antigen receptor (CAR)-engineered lymphocytes for cancer therapy. Expert Opin Biol Ther. 2011;11(7):855-873

51. Plesa G, et al. TCR affinity and specificity requirements for human regulatory T-cell function. Blood. 2012;119(15):3420-3430.

52. Davenport AJ, et al. Chimeric antigen receptor T cells form nonclassical and potent immune synapses driving rapid cytotoxicity. Proc Natl Acad Sci USA. 2018;115(9):E2068-E2076.

53. Jensen MC, et al. Antitransgene rejection responses contribute to attenuated persistence of adoptively transferred CD20/ CD19-specific chimeric antigen receptor redirected T cells in humans. Biol Blood Marrow Transplant. 2010;16(9):1245-1256

54. Pierini A, et al. T cells expressing chimeric antigen receptor promote immune tolerance. JCI Insight. 2017;2(20):92865

55. Nervi B, et al. Factors affecting human T cell engraftment, trafficking, and associated xenogeneic graft-vs-host disease in NOD/ SCID beta2mnull mice. Exp Hematol. 2007;35(12):1823-1838.

56. Zhang N, et al. Regulatory $\mathrm{T}$ cells sequentially migrate from inflamed tissues to draining lymph nodes to suppress the alloimmune response. Immunity. 2009;30(3):458-469.

57. Hoffmann P, et al. Only the CD45RA+ subpopulation of CD4+CD25high T cells gives rise to homogeneous regulatory T-cell lines upon in vitro expansion. Blood. 2006;108(13):4260-4267.

58. Arroyo Hornero R, Betts GJ, Sawitzki B, Vogt K, Harden PN, Wood KJ. CD45RA distinguishes CD4+CD25+CD127-/low TSDR demethylated regulatory $\mathrm{T}$ cell subpopulations with differential stability and susceptibility to tacrolimus-mediated inhibition of suppression. Transplantation. 2017;101(2):302-309.

59. Sadelain M, Brentjens R, Rivière I. The basic principles of chimeric antigen receptor design. Cancer Discov. 2013;3(4):388-398

60. Allan SE, et al. Generation of potent and stable human CD4+ T regulatory cells by activation-independent expression of FOXP3. Mol Ther. 2008;16(1):194-202.

61. Robinson J, Halliwell JA, Hayhurst JD, Flicek P, Parham P, Marsh SG. The IPD and IMGT/HLA database: allele variant databases. Nucleic Acids Res. 2015;43(Database issue):D423-D431. 
62. Lamarche C, et al. Clinical-scale rapid autologous BK virus-specific t cell line generation from kidney transplant recipients with active viremia for adoptive immunotherapy. Transplantation. 2017;101(11):2713-2721.

63. McMurchy AN, Levings MK. Suppression assays with human T regulatory cells: a technical guide. Eur J Immunol. 2012;42(1):27-34.

64. Cooke KR, et al. An experimental model of idiopathic pneumonia syndrome after bone marrow transplantation: I. The roles of minor $\mathrm{H}$ antigens and endotoxin. Blood. 1996;88(8):3230-3239.

65. Hill GR, Crawford JM, Cooke KR, Brinson YS, Pan L, Ferrara JL. Total body irradiation and acute graft-versus-host disease: the role of gastrointestinal damage and inflammatory cytokines. Blood. 1997;90(8):3204-3213.

66. Schneider CA, Rasband WS, Eliceiri KW. NIH Image to ImageJ: 25 years of image analysis. Nat Methods. 2012;9(7):671-675

67. Eliceiri KW, et al. Biological imaging software tools. Nat Methods. 2012;9(7):697-710.

68. Massi D, Franchi A, Pimpinelli N, Laszlo D, Bosi A, Santucci M. A reappraisal of the histopathologic criteria for the diagnosis of cutaneous allogeneic acute graft-vs-host disease. Am J Clin Pathol. 1999;112(6):791-800.

69. Fischer A, et al. Histopathologic features of cutaneous acute graft-versus-host disease in T-cell-depleted peripheral blood stem cell transplant recipients. Am J Dermatopathol. 2015;37(7):523-529.

70. Kanitakis J. The challenge of dermatopathological diagnosis of composite tissue allograft rejection: a review. J Cutan Pathol. 2008;35(8):738-744.

71. Schmittgen TD, Livak KJ. Analyzing real-time PCR data by the comparative C(T) method. Nat Protoc. 2008;3(6):1101-1108. 